\title{
Dynamically Crowded Solutions of Infinitely Thin Brownian Needles
}

\author{
Sebastian Leitmann, ${ }^{1}$ Felix Höfling, ${ }^{2}$ and Thomas Franosch ${ }^{1}$ \\ ${ }^{1}$ Institut für Theoretische Physik, Universität Innsbruck, Technikerstraße 21A, A-6020 Innsbruck, Austria \\ ${ }^{2}$ Fachbereich Mathematik und Informatik, Freie Universität Berlin, Arnimallee 6, 14195 Berlin, Germany
}

(Dated: September 11, 2021)

\begin{abstract}
We study the dynamics of solutions of infinitely thin needles up to densities deep in the semidilute regime by Brownian dynamics simulations. For high densities, these solutions become strongly entangled and the motion of a needle is essentially restricted to a one-dimensional sliding in a confining tube composed of neighboring needles. From the density-dependent behavior of the orientational and translational diffusion, we extract the long-time transport coefficients and the geometry of the confining tube. The sliding motion within the tube becomes visible in the non-Gaussian parameter of the translational motion as an extended plateau at intermediate times and in the intermediate scattering function as an algebraic decay. This transient dynamic arrest is also corroborated by the local exponent of the mean-square displacements perpendicular to the needle axis. Moreover, the probability distribution of the displacements perpendicular to the needle becomes strongly nonGaussian, rather it displays an exponential distribution for large displacements. On the other hand, based on the analysis of higher-order correlations of the orientation we find that the rotational motion becomes diffusive again for strong confinement. At coarse-grained time and length scales, the spatiotemporal dynamics of the needle for the high entanglement is captured by a single freely diffusing phantom needle with long-time transport coefficients obtained from the needle in solution. The time-dependent dynamics of the phantom needle is also assessed analytically in terms of spheroidal wave functions. The dynamic behavior of the needle in solution is found to be identical to needle Lorentz systems, where a tracer needle explores a quenched disordered array of other needles.
\end{abstract}

\section{INTRODUCTION}

Solutions of rod-shaped particles such as filamentous actin (f-actin) [1-4], microtubules [5], xanthan [6], filamentous bacteriophage $\mathrm{fd}[7,8]$, and carbon nanotubes [9] exhibit rich structural and dynamic behavior [10, 11]. Already at the level of a single constituent, the diffusive motion of such an anisotropic particle is much more complex than a spherical one. While the long axis undergoes rotational diffusion, translational diffusion is characterized by a parallel and a slower perpendicular component with respect to the current orientation [12-14]. In solution, rod-shaped particles exhibit different concentration regimes depending on their length $L$ and their diameter $b[15]$. In the dilute regime where the number density $n$ of particles is very small, $n L^{3} \ll 1$, the behavior of a single particle is not affected by its neighbors. The semidilute regime, $n L^{3} \gtrsim 1$, is characterized by a dynamic response due to entanglement effects of the particles with each other and persists as long as the excluded volume of the individual particles is irrelevant, $n \ll 1 / b L^{2}$.

For large aspect ratios $L / b$, the rods can be approximated by infinitely thin needles of length $L$ in the semidilute regime. A remarkable property of such solutions is their trivial ideal-gas-like static structure in striking contrast to their rich dynamic behavior, since no two needles can cross each other. Deep in the semidilute regime, these solutions become dynamically crowded and the dynamics of a single needle is restricted to a sliding motion within a tube formed by its neighbors. As a consequence of this high entanglement, the rotational motion and the translational diffusion perpendicular to the needle slow down drastically, whereas the diffusion along the tube is unaffected. In both theory [15-20] and computer simulations [21-26] the density-dependent scaling behavior of the long-time rotational and perpendicular translational diffusion coefficients have been established and scale with the number density as $n^{-2}$. Computer simulations for two-dimensional toy models have also been performed earlier [27-31] and for a needle in the presence of pointlike obstacles one observes the same scaling laws of the transport coefficients as in three dimensions [29, 30]. In experiments, the transport coefficients of a nanowire diffusing through an array of obstacles have been determined only recently, and the drastic slowing down of transport has been observed [32].

The seminal tube concept for stiff fibers pioneered by Doi and Edwards [33] furthermore reduces the complex many-body dynamics of such solutions on coarse-grained time and length scales to that of a single needle (phantom needle) with very unusual diffusion coefficients. We have shown recently [26] that this striking simplification is valid for the translation-rotation coupling as well as the intermediate scattering function of the geometric center of the needle.

Here, we extend our earlier analysis [26] and consider additional quantities characterizing the dynamics of the needle in solution such as higher-order orientational correlation functions, mean-square displacements, the nonGaussian parameter of the geometric center, and the intermediate scattering function for a needle where each segment contributes to the scattering signal. We also provide analytic formulas for the mentioned quantities in terms of the phantom needle. In particular, for the non-Gaussian parameter in the highly entangled regime, we observe an extended plateau over many decades in 
time which emerges due to the sliding motion of the needle within the confining tube.

\section{STOCHASTIC DYNAMICS}

\section{A. Single needle}

We describe the configuration of a needle by its geometric center $\mathbf{r}$ and its unit vector of orientation, $\mathbf{u}$. The change in position, $\mathrm{d} \mathbf{r}$, and orientation, $\mathrm{d} \mathbf{u}$, of the needle is determined by the following (overdamped) Langevin equations in Itō interpretation $[34,35]$ :

$$
\begin{aligned}
& \mathrm{d} \mathbf{u}=-2 D_{\mathrm{rot}}^{0} \mathbf{u} \mathrm{d} t-\sqrt{2 D_{\mathrm{rot}}^{0}} \mathbf{u} \times \boldsymbol{\xi} \mathrm{d} t, \\
& \mathrm{~d} \mathbf{r}=\left[\sqrt{2 D_{\|}^{0}} \mathbf{u} \mathbf{u}+\sqrt{2 D_{\perp}^{0}}(1-\mathbf{u u})\right] \boldsymbol{\eta} \mathrm{d} t,
\end{aligned}
$$

with rotational diffusion coefficient $D_{\text {rot }}^{0}$ and translational diffusion coefficients for parallel and perpendicular motion, $D_{\|}^{0}$ and $D_{\perp}^{0}$, respectively. The dyadic product $\mathbf{u u}$ acts as a projector onto the long axis $\mathbf{u}$ of the needle and introduces the coupling of translation and rotation. The stochastic nature of the motion is modeled by the independent Gaussian white-noise processes $\boldsymbol{\xi}$ and $\boldsymbol{\eta}$ with zero mean and covariance $\left\langle\xi_{i}(t) \xi_{j}\left(t^{\prime}\right)\right\rangle=\left\langle\eta_{i}(t) \eta_{j}\left(t^{\prime}\right)\right\rangle=$ $\delta_{i j} \delta\left(t-t^{\prime}\right)$.

In computer simulations, we use a discrete fixed Brownian time step $\tau_{\mathrm{B}}$ and implement the Langevin equations [Eq. (1)] by evolving the needle ballistically in the time interval $\Delta t \in\left[0, \tau_{\mathrm{B}}\right]$ via the propagation rules:

$$
\begin{aligned}
\mathbf{u}(t+\Delta t) & =\mathbf{u}(t) \cos (|\boldsymbol{\omega}| \Delta t)+\left(\frac{\boldsymbol{\omega}}{|\boldsymbol{\omega}|} \times \mathbf{u}(t)\right) \sin (|\boldsymbol{\omega}| \Delta t), \\
\mathbf{r}(t+\Delta t) & =\mathbf{r}(t)+\mathbf{v} \Delta t
\end{aligned}
$$

The random pseudovelocities $\boldsymbol{\omega}$ and $\mathbf{v}$ for rotational and translational motion, respectively, are determined at the beginning of every Brownian step according to

$$
\begin{aligned}
& \boldsymbol{\omega}=\sqrt{\frac{2 D_{\mathrm{rot}}^{0}}{\tau_{\mathrm{B}}}}(1-\mathbf{u u}) \mathcal{N}_{\xi}, \\
& \mathbf{v}=\left[\sqrt{\frac{2 D_{\|}^{0}}{\tau_{\mathrm{B}}}} \mathbf{u u}+\sqrt{\frac{2 D_{\perp}^{0}}{\tau_{\mathrm{B}}}}(1-\mathbf{u u})\right] \mathcal{N}_{\eta},
\end{aligned}
$$

where the random variables $\mathcal{N}_{\xi}$ and $\mathcal{N}_{\eta}$ are drawn from a normal distribution with zero mean and unit variance. Both correspond to the Gaussian white-noise processes $\boldsymbol{\xi}$ and $\boldsymbol{\eta}$ in the Langevin equations [Eq. (1)]. The three transport coefficients are not independent and we use the relations $D_{\text {rot }}^{0}=12 D_{\perp}^{0} / L^{2}$ and $D_{\|}^{0}=2 D_{\perp}^{0}$ for a slender rod derived within hydrodynamics [15].

\section{B. Solution of needles}

In the presence of other needles, the needles can no longer diffuse freely since they are not allowed to cross each other. To handle these constraints in the simulation, we employ a pseudo-Brownian scheme $[36,37]$ to account for the hard-core interaction between the needles. The detailed steps for the interaction are outlined in Appendix A. Hydrodynamic interactions are ignored, since they are expected to become unimportant for high aspect ratios [38].

In summary, we subsequently move a single needle and determine possible collisions with other needles during every Brownian time step of duration $\tau_{\mathrm{B}}$. Upon collision, we enforce conservation of energy, momentum, and angular momentum, and the resulting transfer of momentum is directed perpendicular to both orientations of the collision partners (smooth needles). Special care has been taken that at collisions the flow of energy between the rotational and translational degrees of freedom of the moving needle vanishes on average, see Appendix A.

For needle liquids, we use a Brownian time step of $\tau_{\mathrm{B}}=10^{-6} L^{2} / D_{\perp}^{0}$ which is a compromise between choosing very small times to mimic Brownian motion and choosing larger time steps to reach sufficiently long times. In the case of the needle Lorentz system, we move a single tracer needle in a quenched array of other needles with the same hard-core interaction and use $\tau_{\mathrm{B}}=10^{-8} L^{2} / D_{\perp}^{0}$. In both cases, we consider the same densities ranging from infinite dilution to systems deep in the semidilute regime with (reduced) densities over $n^{*}=n L^{3}=10^{3}$ where $n$ is the number of needles per volume.

A single configuration for the needle liquid in a simulation box of size $1.5 \mathrm{~L}$ over $10^{6}$ Brownian time steps takes around $225 \mathrm{CPU}$ hours (Intel@ Core $^{\mathrm{TM}} \mathrm{i} 7-4770 \mathrm{~S} @$ $3.10 \mathrm{GHz})$ for the highest density and we average over at least ten realizations. For the needle Lorentz system we use a simulation box of size $25 L$ and simulate over $10^{10}$ Brownian time steps which takes around $35 \mathrm{CPU}$ hours and we average over $10^{3}$ trajectories for densities $n^{*} \geq 128$.

\section{Phantom needle}

For the needles in solution, the short-time dynamics is described by the short-time diffusion coefficients $D_{\|}^{0}$, $D_{\perp}^{0}$, and $D_{\text {rot }}^{0}$, and differs from the behavior at long times which is characterized by new transport coefficients for translation, $D_{\|}$and $D_{\perp}$, and rotation, $D_{\text {rot }}$. It is instructive to compare the dynamics of a needle in solution to that of a single needle with the emerging long-time diffusion coefficients as input parameters (phantom needle). In computer simulations, this can be easily achieved by evolving the single needle by the Langevin equations [Eq. (1)] with the new transport coefficients for rotation and translation. 

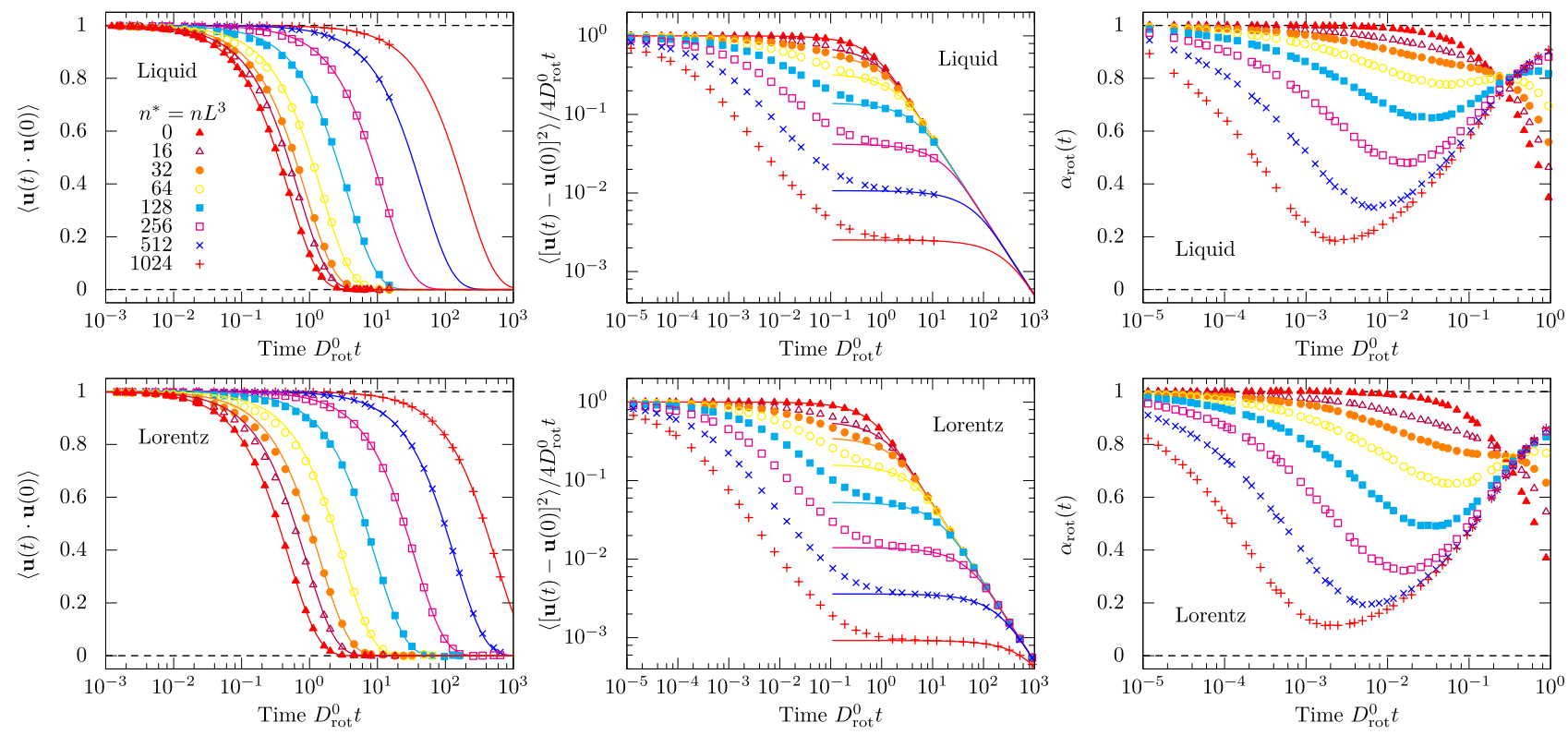

FIG. 1. Rotational diffusion of the needle characterized by the correlation function $\langle\mathbf{u}(t) \cdot \mathbf{u}(0)\rangle$ for the orientation $\mathbf{u}(t)($ left $)$, squared deviation $\left\langle[\mathbf{u}(t)-\mathbf{u}(0)]^{2}\right\rangle=2-2\langle\mathbf{u}(t) \cdot \mathbf{u}(0)\rangle$ in units of the short-time rotational motion $4 D_{\text {rot }}^{0} t$ (middle), and the local exponent $\alpha_{\text {rot }}(t)$ (right). Symbols correspond to simulation results and solid lines represent the phantom needle with long-time rotational diffusion coefficient $D_{\text {rot }}$.

An analytic description is obtained by considering the translationally invariant needle dynamics in space and time in terms of the propagator $G\left(\mathbf{r}, \mathbf{u}, t \mid \mathbf{u}_{0}\right)$. It describes the conditional probability for a displacement $\mathbf{r}$ and a change of orientation from $\mathbf{u}_{0}$ to $\mathbf{u}$ of the needle in lag time $t$. The propagator $G \equiv G\left(\mathbf{r}, \mathbf{u}, t \mid \mathbf{u}_{0}\right)$ obeys the initial condition $G\left(\mathbf{r}, \mathbf{u}, t=0 \mid \mathbf{u}_{0}\right)=\delta(\mathbf{r}) \delta\left(\mathbf{u}, \mathbf{u}_{0}\right)$ such that the needle is oriented along $\mathbf{u}_{0}$ initially. The time evolution of the propagator is then determined by the Smoluchowski-Perrin equation [15, 39]:

$$
\begin{aligned}
\partial_{t} G=D_{\mathrm{rot}} \mathcal{R} & \cdot(\mathcal{R} G) \\
& +\partial_{\mathbf{r}} \cdot\left[D_{\|}\left(\partial_{\mathbf{r}} G\right)-\Delta D(1-\mathbf{u u})\left(\partial_{\mathbf{r}} G\right)\right] .
\end{aligned}
$$

The first term on the right hand side contains the rotational operator $\mathcal{R}=\mathbf{u} \times \partial_{\mathbf{u}}$ and accounts for the change of orientation of the needle in terms of the rotational diffusion coefficient $D_{\text {rot }}$. The second contribution describes the translational diffusion of the needle and couples the diffusional anisotropy $\Delta D=D_{\|}-D_{\perp}$ determined by the parallel $D_{\|}$and the perpendicular diffusion coefficient $D_{\perp}$ to the current orientation $\mathbf{u}(t)$ via the projector $\mathbf{u u}$.

Analytic progress is achieved by considering the spatial Fourier transform $G_{\mathbf{k}}\left(\mathbf{u}, t \mid \mathbf{u}_{0}\right)=\int \mathrm{d}^{3} r e^{-\mathbf{i k} \cdot \mathbf{r}} G\left(\mathbf{r}, \mathbf{u}, t \mid \mathbf{u}_{0}\right)$, which fulfills the following equation:

$$
\begin{aligned}
\partial_{t} G_{\mathbf{k}}=D_{\mathrm{rot}} \mathcal{R} & \cdot\left(\mathcal{R} G_{\mathbf{k}}\right) \\
- & \left\{k^{2} D_{\|}-\Delta D\left[k^{2}-(\mathbf{k} \cdot \mathbf{u})^{2}\right]\right\} G_{\mathbf{k}},
\end{aligned}
$$

with wave vector $\mathbf{k}$ and magnitude $k=|\mathbf{k}|$. We choose a representation in spherical coordinates with polar angle $\theta$ and azimuthal angle $\varphi$ and fix the wave vector $\mathbf{k}=k \mathbf{e}_{z}$ along the $z$-direction and we abbreviate $z=\mathbf{k} \cdot \mathbf{u} / k=\cos (\theta)$. Then, the product $\mathcal{R} \cdot \mathcal{R}$ of the rotational operators reduces to the angular momentum operator $\mathcal{R}_{z, \varphi}^{2}=\partial_{z}\left[\left(1-z^{2}\right) \partial_{z}\right]+\left(1-z^{2}\right)^{-1} \partial_{\varphi}^{2}$ and we obtain

$$
\partial_{t} G_{k}=D_{\text {rot }} \mathcal{R}_{z, \varphi}^{2} G_{k}-k^{2}\left[D_{\|}-\Delta D\left(1-z^{2}\right)\right] G_{k} .
$$

This partial differential equation is solved by a separation of variables and the full solution [40] is obtained as an expansion in terms of spheroidal wave functions $\mathrm{Ps}_{n}^{m}$ of degree $n$ and order $m$ :

$$
\begin{array}{r}
G_{k}\left(z, \varphi, t \mid z_{0}, \varphi_{0}\right)=\sum_{m=-\infty}^{\infty} \sum_{n=m}^{\infty} \frac{2 n+1}{4 \pi} \frac{(n-m) !}{(n+m) !} \times \\
\times \operatorname{Ps}_{n}^{m}\left(z, \gamma^{2}\right) \operatorname{Ps}_{n}^{m}\left(z_{0}, \gamma^{2}\right) e^{\mathrm{i} m\left(\varphi-\varphi_{0}\right)} e^{-\Gamma_{n}^{m} t}
\end{array}
$$

with real parameter $\gamma^{2}:=k^{2} \Delta D / D_{\text {rot }} \geq 0$ and characteristic decay constants $\Gamma_{n}^{m} \equiv \Gamma_{n}^{m}\left(\gamma^{2}\right) \geq 0$. The exact relations for both parameters are determined as solutions of the spheroidal wave equation $[41,42]$

$$
\begin{aligned}
\partial_{z}[(1 & \left.\left.-z^{2}\right) \partial_{z} \mathrm{Ps}_{n}^{m}\right] \\
& +\left[\lambda_{n}^{m}\left(\gamma^{2}\right)+\gamma^{2}\left(1-z^{2}\right)-\frac{m^{2}}{1-z^{2}}\right] \operatorname{Ps}_{n}^{m}=0
\end{aligned}
$$

with spheroidal eigenvalue $\lambda_{n}^{m} \equiv \lambda_{n}^{m}\left(\gamma^{2}\right)$. The decay constants are related to the spheroidal eigenvalue via $\Gamma_{n}^{m}=D_{\|} k^{2}+D_{\text {rot }} \lambda_{n}^{m}$.

The propagator $G_{k}$ [Eq. (7)] contains the full information about the dynamics of the phantom needle and can be used to obtain explicit expressions for the quantities of interest. 


\section{TRANSPORT BEHAVIOR}

\section{A. Rotational diffusion}

We first consider the effect of the dynamic crowding on the time-dependent orientation $\mathbf{u}(t)$. A simple quantity which encodes the topological constraints imposed by the neighboring needles is given by the time-dependent orientational correlation function $\langle\mathbf{u}(t) \cdot \mathbf{u}(0)\rangle$. The angle brackets denote an ensemble average over all moving needles and configurations and the initial orientation $\mathbf{u}(0)$ is uniformly distributed over the sphere.

In the absence of other needles, the correlation function decays exponentially, $\langle\mathbf{u}(t) \cdot \mathbf{u}(0)\rangle=\exp \left(-2 D_{\operatorname{rot}}^{0} t\right)$, and the time scale for the decay is determined by the rotational diffusion coefficient $D_{\text {rot }}^{0}$ [15] [Fig. 1]. With increasing needle density $n^{*}$, the initial orientation $\mathbf{u}(0)$ persists for longer times since the needle can no longer rotate freely due to the topological constraints imposed by its neighbors. The time-dependent long-time relaxation of the correlation function is again captured by an exponential decay $\langle\mathbf{u}(t) \cdot \mathbf{u}(0)\rangle=\exp \left(-2 D_{\text {rot }} t\right)$ and the time scale for the decay encodes the long-time rotational diffusion coefficient $D_{\text {rot }}$. Deviations from the rotational motion of the phantom needle are present at intermediate times where the needle explores its close environment and they become visible for small densities where the concept of a confining tube is not fully applicable.

The time-dependent behavior preceding the exponential decay at long times in the correlation function $\langle\mathbf{u}(t) \cdot \mathbf{u}(0)\rangle$ can be analyzed more closely by the directly related quantity of the squared distance of the time-dependent orientation $\mathbf{u}(t)$ and the initial one $\mathbf{u}(0)$ : $\left\langle[\mathbf{u}(t)-\mathbf{u}(0)]^{2}\right\rangle=2-2\langle\mathbf{u}(t) \cdot \mathbf{u}(0)\rangle$. For a freely diffusing needle, the change in orientation is described by ordinary diffusion in two dimensions with diffusion constant $D_{\text {rot }}^{0}$ as long as the needle has not rotated significantly $\left(t \ll 1 / D_{\text {rot }}^{0}\right)$ :

$$
\left\langle[\mathbf{u}(t)-\mathbf{u}(0)]^{2}\right\rangle=4 D_{\text {rot }}^{0} t+\mathcal{O}\left(D_{\text {rot }}^{0} t\right)^{2} .
$$

The drastic slowing down of rotational diffusion with increasing density of neighboring needles is exemplified by considering the squared distance of orientations in units of the short-time diffusion of the freely diffusing needle [Fig. 1]. For times $t \lesssim 1 / D_{\text {rot }}^{0}$ the rotational motion becomes strongly suppressed for high needle densities and approaches an intermediate plateau at times $t \gtrsim 1 / D_{\text {rot }}^{0}$ where the squared distance increases linearly with long-time rotational diffusion coefficient $D_{\text {rot }}$. This plateau with the following crossover to the exponential decay of the correlation function offers a direct way to determine $D_{\text {rot }}$ since it separates the short-time behavior, where the needle explores its environment, from the emerging stochastic motion of the phantom needle at long times. The extracted longtime rotational diffusion coefficient follows the scaling law $D_{\text {rot }} \sim\left(n^{*}\right)^{-2}$ [Fig. 2] where we normalized the density
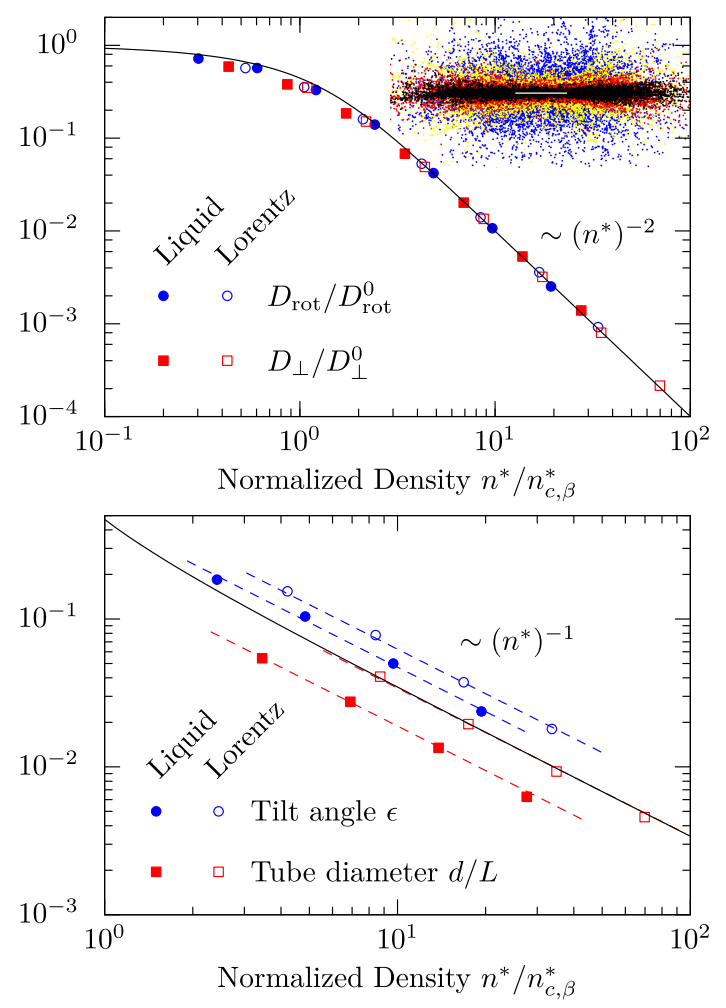

FIG. 2. Top: Longtime diffusion coefficients for rotation, $D_{\text {rot }}$, and perpendicular diffusion $D_{\perp}$ as a function of the normalized density $n^{*} / n_{c, \beta}^{*}$ with respect to the rotational $(\beta=\operatorname{rot})$ and translational motion $(\beta=\perp)$ for needle liquids as well as needle Lorentz systems. The solid line corresponds to the theoretical prediction [Eq. (17)]. Inset (top): Distribution of the geometric centers of the needle for densities $n^{*}=128$ (blue), 256 (yellow), 512 (red), and 1024 (black) up to times $L^{2} / 2 D_{\perp}^{0}$. The initial position and orientation of the needle are indicated by the white needle in the center. For increasing density, the motion of the needle becomes more and more directed along the initial orientation as anticipated by the tube theory. Bottom: Tilt angle $\epsilon$ and tube diameter $d$ with respect to the normalized density. The solid line represents the theoretical prediction for the localization length [Eq. (19)]. Figure is adjusted from Ref. [26].

by the inverse of the respective prefactor $n_{c \text {,rot }}^{*} \approx 53$ and $n_{c, \text { rot }}^{*} \approx 30$ for needle liquids and needle Lorentz systems, respectively. The scaling behavior has been predicted by theory [16, 19] and also confirmed by earlier computer simulations [21, 23, 24].

The needle explores its close environment in the time window $t \lesssim 1 / D_{\text {rot }}^{0}$, which contains information about the geometry of the confining tube. We measure the suppression of the rotational diffusion by the local exponent

$$
\alpha_{\text {rot }}(t)=\frac{\mathrm{d} \ln \left(\left\langle[\mathbf{u}(t)-\mathbf{u}(0)]^{2}\right\rangle\right)}{\mathrm{d} \ln (t)}=-t \frac{\mathrm{d}\langle\mathbf{u}(t) \cdot \mathbf{u}(0)\rangle / \mathrm{d} t}{1-\langle\mathbf{u}(t) \cdot \mathbf{u}(0)\rangle} .
$$

With increasing density, $\alpha_{\text {rot }}(t)$ becomes more and more suppressed at intermediate times and we expect a tran- 

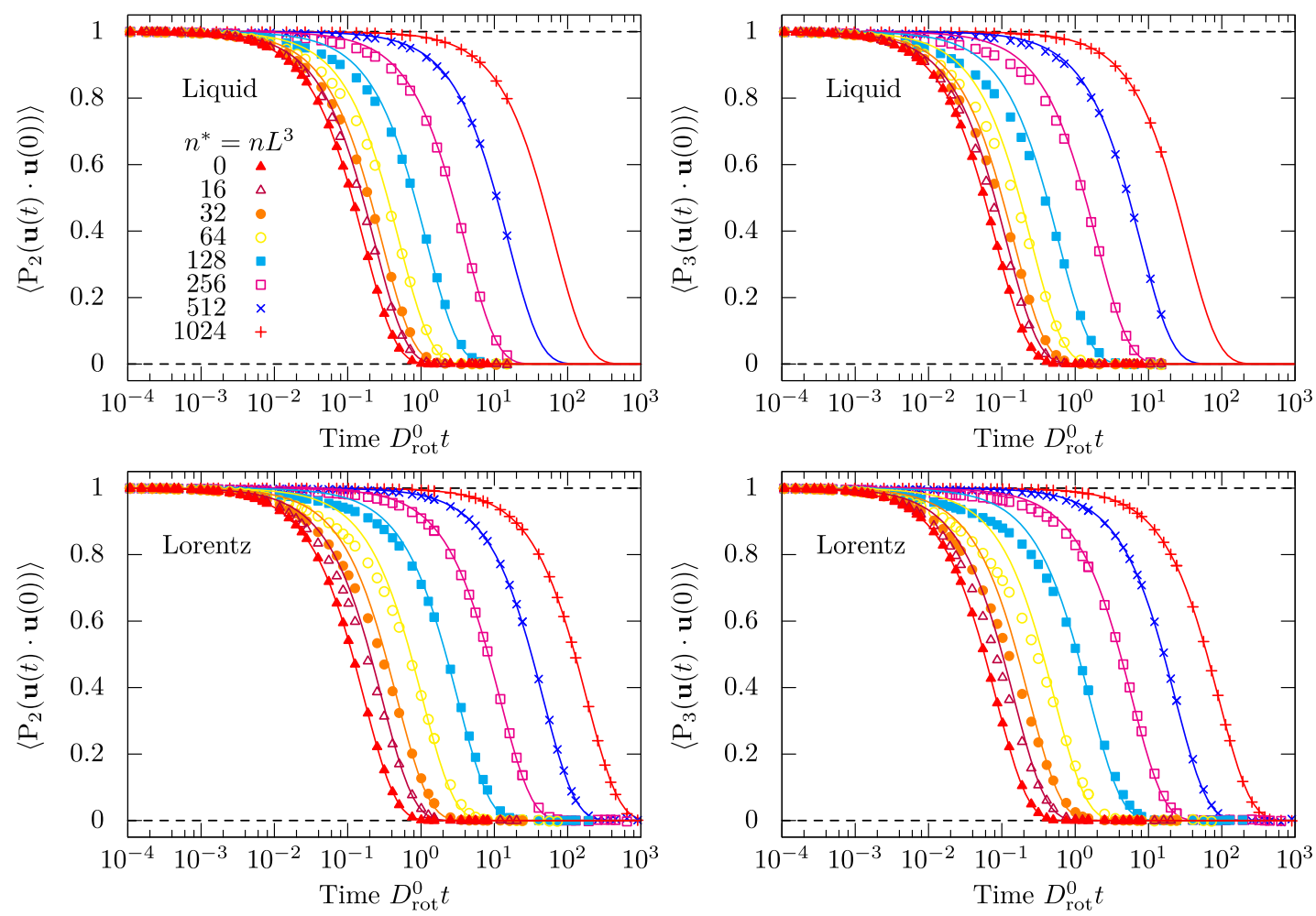

FIG. 3. Time-dependent higher-order orientational correlation functions $\left\langle\mathrm{P}_{\ell}(\mathbf{u}(t) \cdot \mathbf{u}(0))\right\rangle$ for $\ell=2$ (left) and $\ell=3$ (right) at different densities $n^{*}$ for needle liquids as well as needle Lorentz systems. Density increases from left to right. Symbols represent results from computer simulations and solid lines represent the analytic solution $\exp \left[-\ell(\ell+1) D_{\text {rot }} t\right]$ of a freely diffusing phantom needle with long-time rotational diffusion coefficient $D_{\text {rot }}$.

sient dynamic arrest of the rotational motion by going beyond the densities considered here [Fig. 1]. The time scale for the onset of the relaxation of the arrest is set by the density-independent time for the needle to diffuse along its long axis $L^{2} / D_{\|}^{0}$, leading to a collapse of the data. For long times, the squared deviation of the orientations on the sphere saturates $\lim _{t \rightarrow \infty}\left\langle[\mathbf{u}(t)-\mathbf{u}(0)]^{2}\right\rangle=2$ irrespective of the density, resulting in an apparent common intersection point and a vanishing of the local exponent $\alpha_{\text {rot }}(t \rightarrow \infty)=0$.

In principle, the tilt angle $\epsilon$ of the needle should become manifest as a plateau of order $\epsilon^{2}$ in the deviations of the orientational correlation function from its initial value at intermediate times: $1-\langle\mathbf{u}(t) \cdot \mathbf{u}(0)\rangle=1-\cos (\epsilon)$. However, since the plateau is not very well pronounced we use a more robust determination in terms of the previously defined local exponent for the rotational motion [Eq. (10)]. Here, we define the tilt angle $\epsilon$ via the orientational correlation function $\left\langle\mathbf{u}\left(\tau_{\epsilon}\right) \cdot \mathbf{u}(0)\right\rangle=\cos (\epsilon)$ at time $\tau_{\epsilon}$, at which the local exponent $\alpha_{\perp}$ becomes minimal [Fig. 1]. In our data such a minimum in the local exponent $\alpha_{\text {rot }}$ emerges for times $t<1 / D_{\text {rot }}^{0}$ and for densities $n^{*} \geq 64$ and becomes more and more pronounced for increasing density. For the tilt angle of the needle in the confining tube, we also recover the predicted scaling behavior $\epsilon \sim\left(n^{*}\right)^{-1}$ [15] (Fig. 2 reproduced from Ref. [26]).
With the extracted long-time rotational diffusion coefficient $D_{\text {rot }}$, we can directly compare the dynamics in needle liquids and needle Lorentz systems to the dynamics of a phantom needle in terms of higher-order orientational correlation functions

$$
\left\langle\mathrm{P}_{\ell}(\mathbf{u}(t) \cdot \mathbf{u}(0))\right\rangle=\exp \left[-\ell(\ell+1) D_{\text {rot }} t\right] .
$$

where $\mathrm{P}_{\ell}(\cdot)$ denotes the Legendre polynomial of degree $\ell$ [15]. From the full solution of the propagator $G_{k}$ [Eq. (7)], the preceding relation [Eq. (11)] can be derived in the following way: For the rotation, we are not interested in the spatial dynamics and we set $k=0$ in the expression for the propagator of the phantom needle $G_{0} \equiv G_{k=0}[$ Eq. (7)]. Then, the real parameter $\gamma^{2}:=k^{2} \Delta D / D_{\text {rot }}$ vanishes and the spheroidal wave functions reduce to the associated Legendre polynomials $\mathrm{Ps}_{n}^{m}(z, 0)=\mathrm{P}_{n}^{m}(z)$ with eigenvalue $\lambda_{n}^{m}(0)=n(n+1)$. We express the summands in spherical harmonics $Y_{n m}(\cdot)$ and obtain with the addition theorem $(2 n+1) \mathrm{P}_{n}\left(\mathbf{u} \cdot \mathbf{u}_{0}\right)=$ $4 \pi \sum_{m=-n}^{n} Y_{n m}\left(\mathbf{u}_{0}\right) Y_{n m}^{*}(\mathbf{u})$ the propagator for the rotational dynamics [19]:

$$
G_{0}\left(\mathbf{u}, t \mid \mathbf{u}_{0}\right)=\sum_{n=0}^{\infty} \frac{2 n+1}{4 \pi} e^{-n(n+1) D_{\mathrm{rot}} t} \mathrm{P}_{n}\left(\mathbf{u} \cdot \mathbf{u}_{0}\right) .
$$

The correlation functions [Eq. (11)] then follow by averaging over all initial orientations and integrating over all 

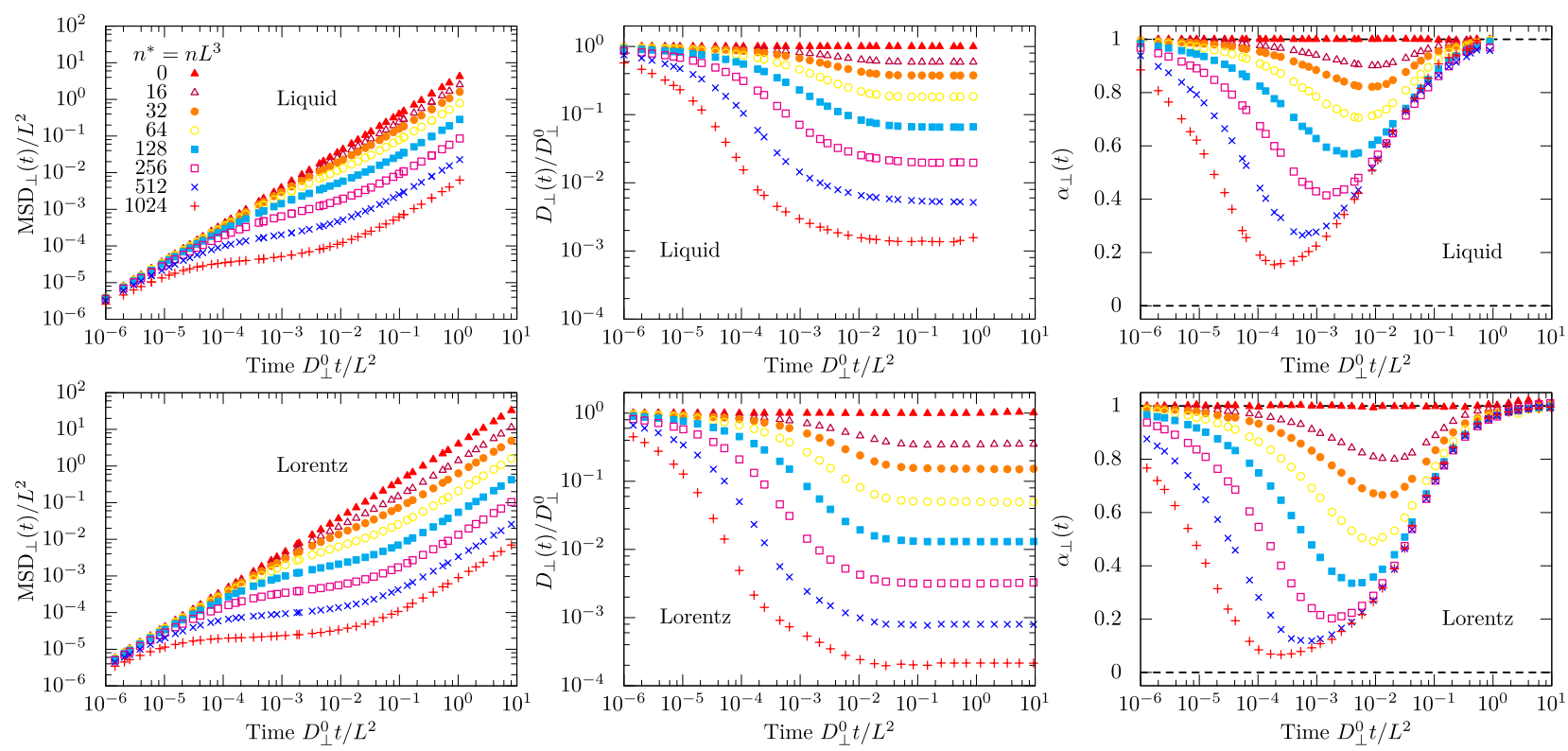

FIG. 4. Perpendicular translational diffusion measured in a coordinate frame fixed to the needle characterized by the meansquare displacement $\mathrm{MSD}_{\perp}(t)$ (left), the diffusion coefficient $D_{\perp}(t)$ (middle), and the local exponent $\alpha_{\perp}(t)$ (right). Density increases from top to bottom. Symbols correspond to simulation results.

final orientations:

$$
\left\langle\mathrm{P}_{\ell}(\mathbf{u}(t) \cdot \mathbf{u}(0))\right\rangle=\int \mathrm{d}^{2} u \int \frac{\mathrm{d}^{2} u_{0}}{4 \pi} \mathrm{P}_{\ell}\left(\mathbf{u} \cdot \mathbf{u}_{0}\right) G_{0}\left(\mathbf{u}, t \mid \mathbf{u}_{0}\right) .
$$

The measured correlation functions of orders two and three show stronger deviations at smaller densities than the first-order orientational correlation function [Fig. 3]. However, for high entanglement, the dynamics of the phantom needle and the needle liquid and needle Lorentz systems are indistinguishable. Thus, on coarse-grained time scales and for the strong entanglement the pure orientational motion is described by simple diffusion on a sphere.

\section{B. Translational diffusion}

We first discuss the translational dynamics in a coordinate frame comoving with the needle. The coordinate frame is defined by three orthonormal basis vectors: one aligned parallel to the long axis, $\mathbf{u}(t)$, and two perpendicular directions denoted by $\mathbf{u}_{1}(t)$ and $\mathbf{u}_{2}(t)$. The time evolution of $\mathbf{u}(t)$ is described in Eq. (2), whereas the perpendicular directions follow by rotating

$$
\begin{aligned}
\mathbf{u}_{i}(t+\Delta t)= & \mathbf{u}_{i}(t) \cos (|\boldsymbol{\omega}| \Delta t)+\left(\frac{\boldsymbol{\omega}}{|\boldsymbol{\omega}|} \times \mathbf{u}_{i}(t)\right) \sin (|\boldsymbol{\omega}| \Delta t) \\
& +\frac{\boldsymbol{\omega}}{|\boldsymbol{\omega}|}\left(\frac{\boldsymbol{\omega}}{|\boldsymbol{\omega}|} \cdot \mathbf{u}_{i}(t)\right)[1-\cos (|\boldsymbol{\omega}| \Delta t)] .
\end{aligned}
$$

Then, the parallel and perpendicular displacements in the comoving frame with respect to the displacement of the geometric center are obtained by $\Delta r_{\|}(t)=$ $\int_{0}^{t} \mathrm{~d} t^{\prime} \mathbf{v}\left(t^{\prime}\right) \cdot \mathbf{u}\left(t^{\prime}\right)$ and $\Delta r_{i}(t)=\int_{0}^{t} \mathrm{~d} t^{\prime} \mathbf{v}\left(t^{\prime}\right) \cdot \mathbf{u}_{i}\left(t^{\prime}\right)$, respectively.

Since we consider infinitely thin smooth needles, the collisions do not influence the parallel motion and the mean-square displacement parallel to the long axis, $\operatorname{MSD}_{\|}(t)=\left\langle\Delta r_{\|}^{2}(t)\right\rangle$, is unaffected by the dynamic crowding and it is diffusive at all times with long-time diffusion coefficient $D_{\|}=D_{\|}^{0}$ :

$$
\operatorname{MSD}_{\|}(t)=2 D_{\|}^{0} t
$$

The mean-square displacement perpendicular to the long axis, $\mathrm{MSD}_{\perp}(t)=\left\langle\Delta r_{1}^{2}(t)\right\rangle+\left\langle\Delta r_{2}^{2}(t)\right\rangle$, shows a strong suppression with increasing density at intermediate times due to the caging by neighboring needles with a crossover to ordinary diffusion for long times [Fig. 4]. We define the time-dependent diffusion coefficient perpendicular to the needle by the derivative

$$
D_{\perp}(t)=\frac{1}{4} \frac{\mathrm{d}}{\mathrm{d} t} \operatorname{MSD}_{\perp}(t) .
$$

Then, the regime of ordinary diffusion at long times is reflected by a plateau, which encodes the perpendicular long-time diffusion coefficient $D_{\perp}:=D_{\perp}(t \rightarrow$ $\infty)$ [Fig. 4]. The extracted diffusion coefficient $D_{\perp}$ exhibits the same scaling behavior [Fig. 2] as the rotational long-time diffusion coefficient, $D_{\perp} \sim\left(n^{*}\right)^{-2}$, and the scaling behavior also confirms the theoretical predictions [18, 20, 43]. For the normalization of the density we used the square root of the prefactor of the scaling behavior, which reads $n_{\mathrm{c}, \perp}^{*} \approx 37$ and $n_{\mathrm{c}, \perp}^{*} \approx 15$ for needle liquids and needle Lorentz systems, respectively. The 

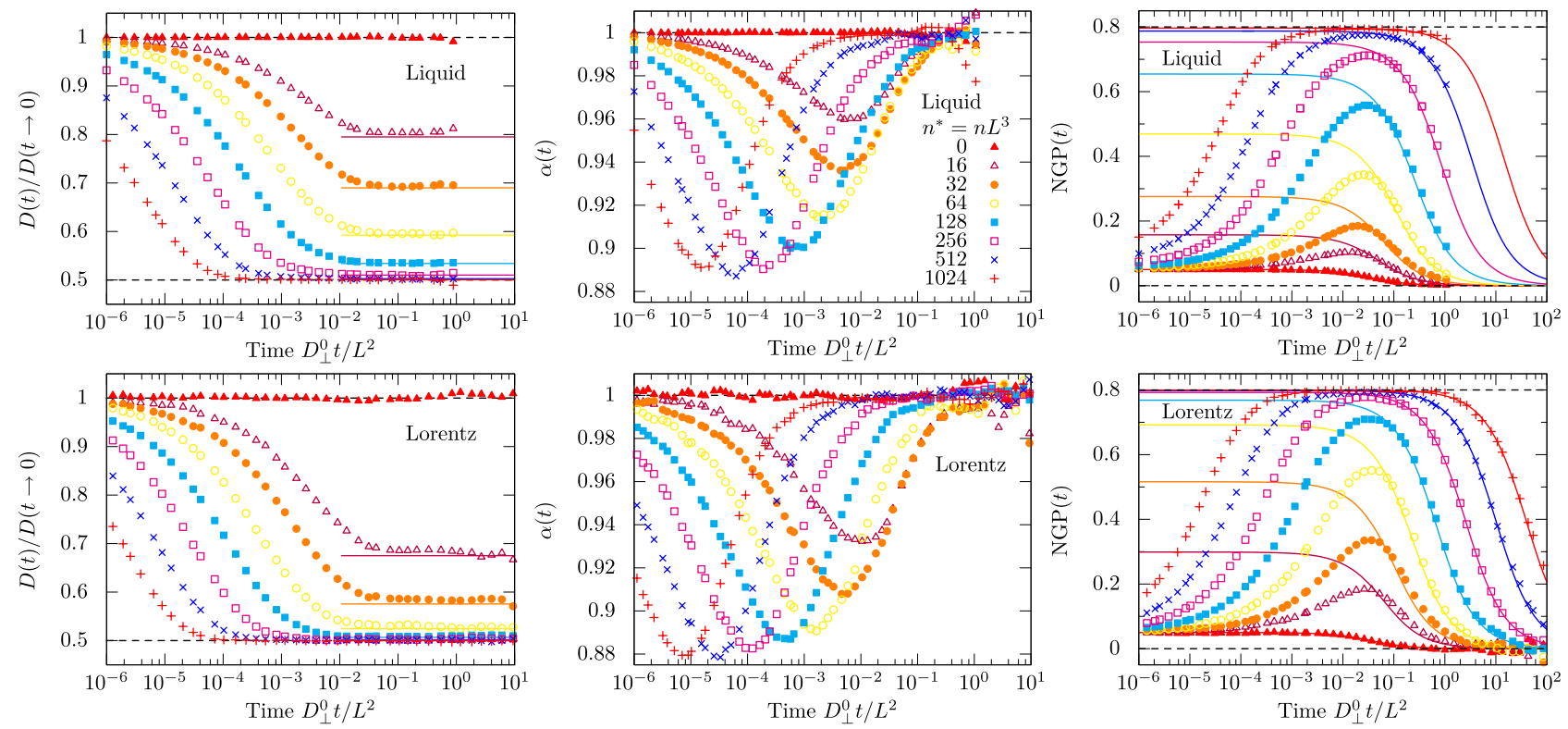

FIG. 5. Time-dependent diffusion coefficient of the geometric center $D(t)$ (left), the local exponent $\alpha(t)$ (middle), and non-Gaussian parameter $\mathrm{NGP}(t)$ (right) at different densities for needle liquids as well as needle Lorentz systems. Symbols correspond to simulation results and lines represent the behavior of the phantom needle.

density-dependent behavior of the diffusion coefficient $D_{\perp}$ is captured by a theoretical prediction for solutions of nonrotating infinitely thin needles $[20,43]$ where the suppression of the diffusion coefficient can be expressed in terms a self-consistent equation of the form

$$
D_{\perp} / D_{\perp}^{0}=\frac{1}{1+\left(D_{\perp}^{0} / 2 D_{\perp}\right)^{1 / 2} n^{*} \lambda_{\perp}\left(D_{\perp} / 2 D_{\perp}^{0}\right)},
$$

where $\lambda_{\perp}(\cdot)$ denotes a complicated monotonic function and we used $D_{\|}^{0}=2 D_{\perp}^{0}$. An explicit expression of $\lambda_{\perp}(\cdot)$ is given in Eq. (A16) of Ref. [43]. For strong suppression, the asymptotic behavior of the self-consistent equation [Eq. (17)] is obtained as $D_{\perp} / D_{\perp}^{0} \sim 36 \pi\left(n^{*}\right)^{-2}$, which defines the normalization $n_{c, \perp}^{*}=6 \sqrt{\pi}$ for comparison with our simulation results.

Information about the geometry of the confining tube is contained in the time-dependent suppression of the perpendicular mean-square displacement $\operatorname{MSD}_{\perp}(t)$ and can be extracted by considering the local exponent

$$
\alpha_{\perp}(t)=\frac{\mathrm{d} \ln \left(\mathrm{MSD}_{\perp}(t)\right)}{\mathrm{d} \ln (t)}=\frac{2 D_{\perp}(t) t}{\mathrm{MSD}_{\perp}(t)},
$$

similar to the tilt angle for the rotational motion [Eq. (10)]. With increasing density, the local exponent becomes more and more suppressed at intermediate times and nearly vanishes for the highest density considered in the Lorentz system. Similar to the local exponent for the rotation $\alpha_{\text {rot }}(t)$ [Eq. (10)], we expect a transient dynamic arrest at intermediate times by further increasing the density. The time scale for the increase of the local exponent is again set by the time scale for the parallel diffusion leading to a collapse of the data for high entanglement. We use the time $\tau_{\perp}$ corresponding to the minimum in $\alpha_{\perp}(t)$ to define the tube diameter $d$ via $\operatorname{MSD}_{\perp}\left(\tau_{\perp}\right)=d^{2}$, which exhibits the predicted scaling, $d \sim\left(n^{*}\right)^{-1}[20,44,45]$ [Fig. 2]. We also compare the tube diameter $d$ obtained from our simulations to a theoretical prediction of the tube localization length for nonrotating infinitely thin needles. Just recently, the densitydependent behavior of the tube-localization length $r_{\text {loc }}$ has been extended to all densities [44, 45] and is given by the self-consistent equation

$$
\frac{L^{2}}{r_{\mathrm{loc}}^{2}}=\frac{\pi n^{*}}{4 \sqrt{2}} \frac{L^{2}}{r_{\mathrm{loc}}^{2}} \lambda_{\mathrm{loc}}\left(L / r_{\mathrm{loc}}\right)
$$

for nonrotating needles performing only perpendicular diffusion. The function $\lambda_{\text {loc }}(x)=\left[x-I_{1}(2 x)+\right.$ $\left.L_{1}(2 x)\right] / 2 x^{2}$ is defined in terms of the first modified Bessel function $I_{1}(\cdot)$ and the first modified Struve function $L_{1}(\cdot)$. For very small localization lengths $r_{\text {loc }} / L \ll$ 1 , the self-consistent equation reduces to the previously known scaling behavior $r_{\text {loc }} / L \sim 8 \sqrt{2} / \pi n^{*}[20,43]$. The predicted tube-localization length with normalization $n_{c, \perp}^{*}=6 \sqrt{\pi}$ nicely captures the range of tube diameters $d$ for needle liquids and needle Lorentz systems [Fig. 2]. Interestingly, the prediction coincides with our result of the tube diameter for the needle Lorentz system.

The next interesting quantity for the translational diffusion of the needle is given by the mean-square displacement of the geometric center in the laboratory fixed frame, $\operatorname{MSD}(t)=\left\langle|\Delta \mathbf{r}(t)|^{2}\right\rangle$. We discuss the time dependence of the mean-square displacement via the time- 

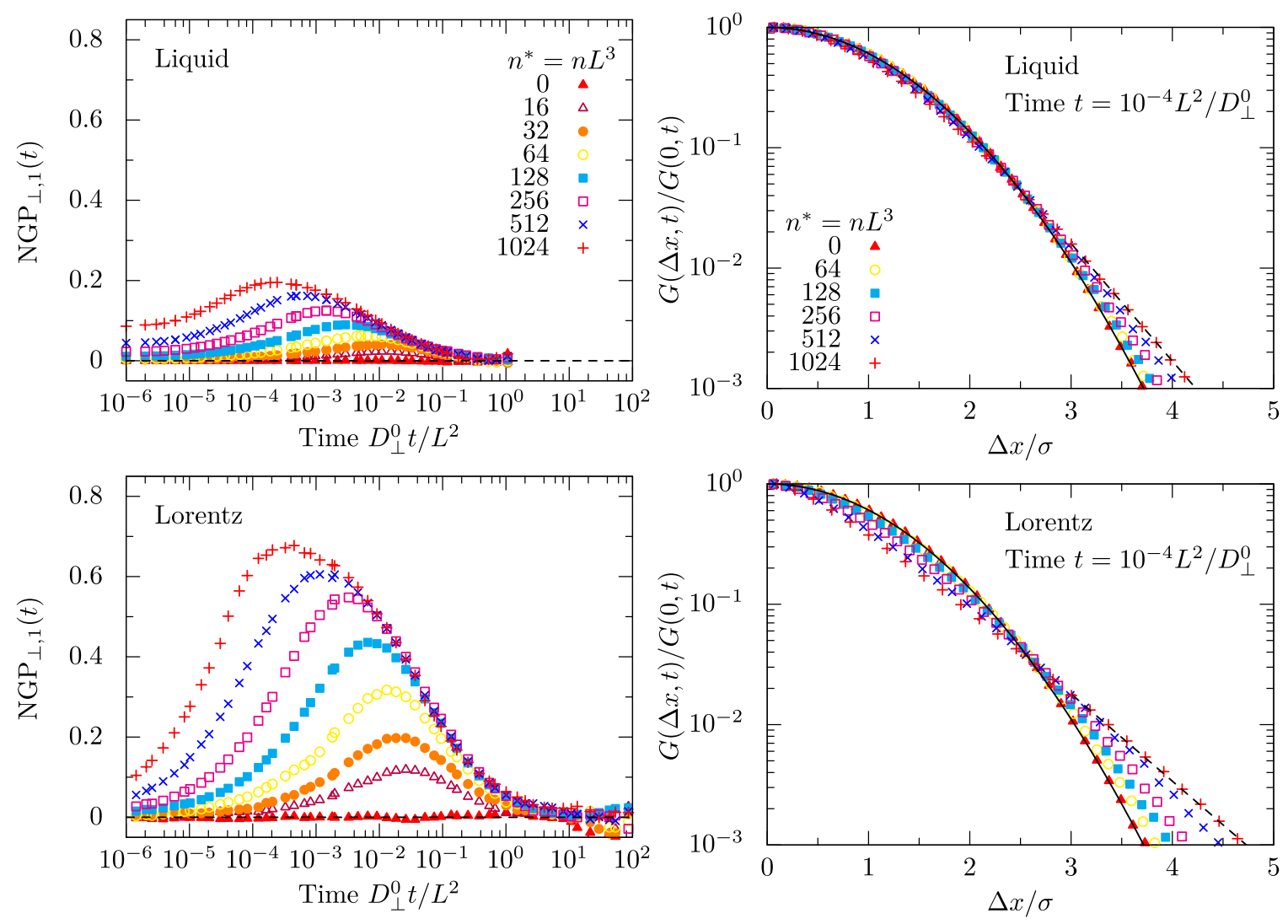

FIG. 6. Left: Non-Gaussian parameter in the comoving frame along a direction perpendicular to the needle axis for needle liquids as well as needle Lorentz systems. Right: Probability distribution $G(\Delta x, t)$ of the displacements $\Delta x$ at time $t=10^{-4} L^{2} / D_{\perp}^{0}$ along one direction perpendicular to the needle axis measured in the comoving frame. Symbols correspond to computer simulations. The displacement $\Delta x$ is normalized by the standard deviation $\sigma$ of the respective distribution $G(\Delta x, t)$. The solid line represents the Gaussian behavior, and the dashed lined indicates an exponential decay for large displacements.

dependent diffusion coefficient [Fig. 5]

$$
D(t)=\frac{1}{6} \frac{\mathrm{d}}{\mathrm{d} t} \operatorname{MSD}(t) .
$$

For short times, the diffusion coefficient is determined by the short-time diffusion coefficients for translation: $D(t \rightarrow 0)=\left(D_{\|}^{0}+2 D_{\perp}^{0}\right) / 3$. In the presence of other needles, the diffusion coefficient decreases over time due to the suppression of the perpendicular needle motion [Fig. 5]. For strong entanglement, the contributions from translational diffusion perpendicular to the needle vanish in comparison to the parallel one, and the longtime diffusion coefficient $D:=D(t \rightarrow \infty)$ approaches its limiting value $D_{\|} / 3$. On coarse-grained time scales, the phantom needle again captures the dynamics.

The translational dynamics of the geometric center can also be discussed in terms of the local exponent

$$
\alpha(t)=\frac{\mathrm{d} \ln (\operatorname{MSD}(t))}{\mathrm{d} \ln (t)}=\frac{2 D(t) t}{\operatorname{MSD}(t)} .
$$

For increasing density, the crossover regime shifts to earlier times [Fig. 5] as anticipated from the diffusion coefficient $D(t)$. The local exponent exhibits a lower bound for the considered densities and by scaling the time with the squared density, the data collapse for densities $n^{*} \gtrsim 256$. Since the parallel diffusion of the needle is unaffected by neighboring needles, the time-dependent behavior of the local exponent $\alpha(t)$ is quite different from that found in models of porous media [46-48] and glass- forming systems [49-51], which exhibit anomalous diffusion and a localization transition.

Deviations from ordinary diffusion are quantified by the non-Gaussian parameter defined via $[52,53]$

$$
\operatorname{NGP}(t)=\frac{3}{5} \frac{\operatorname{MQD}(t)}{\operatorname{MSD}(t)^{2}}-1,
$$

with mean-quartic displacement $\operatorname{MQD}(t)=\left\langle|\Delta \mathbf{r}(t)|^{4}\right\rangle$ of the geometric needle center. For the phantom needle it follows via a time-dependent perturbation theory with Eq. (6) [54] (for a derivation in terms of spheroidal wave functions see Ref. [55] and Appendix B):

$$
\operatorname{MQD}(t)=60 D^{2} t^{2}+\frac{8(\Delta D)^{2}}{27 D_{\mathrm{rot}}^{2}}\left[6 D_{\mathrm{rot}} t-1+e^{-6 D_{\mathrm{rot}} t}\right] .
$$

Thus, for the non-Gaussian parameter of the phantom 
needle, it follows that

$$
\operatorname{NGP}(t)=\frac{8}{45}\left(\frac{\Delta D / D}{6 D_{\mathrm{rot}} t}\right)^{2}\left[6 D_{\mathrm{rot}} t-1+e^{-6 D_{\mathrm{rot}} t}\right] .
$$

In particular, $\operatorname{NGP}(t)$ is nonvanishing for anisotropic diffusion and it approaches zero only algebraically $\operatorname{NGP}(t)=\mathcal{O}\left(t^{-1}\right)$ as anticipated by the central limit theorem.

In the presence of other needles the short-time nonGaussian parameter is determined by the short-time diffusion coefficients and is given by $\operatorname{NGP}(t \rightarrow 0)=1 / 20$ for a slender rod. With increasing density of needles, the rotational and the translational diffusion coefficient perpendicular to the needle axis become more and more suppressed. In particular, the perpendicular diffusion coefficient becomes negligible in comparison to the parallel one which is not affected by the disorder. Thus, for the phantom needle, the diffusional anisotropy $\Delta D$ and diffusion coefficient $D$ are purely determined by the parallel diffusion coefficient with $\Delta D=D_{\|}$and $D=D_{\|} / 3$. In this case and for times where the rotational motion is small, the non-Gaussian parameter [Eq. (24)] of the phantom needle reduces to

$$
\operatorname{NGP}(t)=\frac{4}{5}\left[1-2 D_{\text {rot }} t+\mathcal{O}\left(D_{\text {rot }} t\right)^{2}\right],
$$

and the purely translational motion parallel to the long axis of the phantom needle emerges as a plateau $4 / 5$ as long as the phantom needle has not rotated significantly. This plateau is observed in our data for the highest densities considered for the needle in solution [Fig. 5]. There, the needle probes its confining tube of diameter $d$ at time scale $d^{2} / D_{\perp}^{0} \sim 1 /\left(n^{*}\right)^{2}$, whereas the long-time rotational relaxation becomes relevant only for times $1 / D_{\text {rot }} \sim\left(n^{*}\right)^{2}$. Again, we find that the phantom needle captures the time-dependent behavior at high densities and on coarse-grained time scales, once the needle has explored its initial tube and rotates with long-time diffusion coefficient $D_{\text {rot }}$.

The contributions to the NGP $(t)$ discussed above arise essentially from the anisotropy of the diffusion, masking actual non-Gaussian behavior in the individual Cartesian components. Since the needle can freely diffuse along its long axis irrespective of the density of neighboring needles, the non-Gaussian parameter of $\Delta r_{\|}(t)$, measured in the comoving frame along the needle axis vanishes. This is in striking contrast to the motion perpendicular to the needle axis which exhibits strong deviations from ordinary diffusion as indicated by the non-Gaussian parameter $\mathrm{NGP}_{\perp, 1}(t)$ of the displacement $\Delta x \equiv \Delta r_{1}$ of the needle perpendicular to its long axis along one direction in the comoving frame [Fig. 6 (left)]. Here, we further investigate this scenario in terms of the probability distribution $G(\Delta x, t)$ of the displacements $\Delta x \equiv \Delta r_{1}$, which we evaluate at time $t=10^{-4} L^{2} / D_{\perp}^{0}$ to minimize the influence of the parallel motion of the needle as indicated by the local exponent $\alpha_{\perp}(t)$ [Fig. 4 (right)]. Hence, the needle only probes the confining tube due to the neighboring needles.

The probability distribution $G(\Delta x, t)$ develops significant deviations from a Gaussian with increasing density of the needles [Fig. 6 (right)]. In particular for the needle Lorentz system and for large displacements, the distribution $G(\Delta x, t)$ approaches an exponential, which may be interpreted as a constant effective restoring force $f=-k_{\mathrm{B}} T \mathrm{~d} \ln [G(\Delta x, t)] / \mathrm{d}(\Delta x)$ on the needle due to the confining tube. This scenario has been anticipated theoretically just recently for solutions of infinitely thin needles where the full tube confinement potential has been constructed for the first time [45]. The tube confinement potential exhibits a strongly anharmonic character and thus a distribution of tube diameters or transverse localization lengths on intermediate scales. Experimentally, this scenario has also been observed for entangled solutions of semiflexible polymers where the tube width distribution is not harmonic due to the existence of stretched tails and a displacement-independent effective restoring force $[56,57]$. While these anharmonic displacements are also observed for needle liquids, they are much less pronounced in comparison to needle Lorentz systems due to the existence of constraint release processes.

Such exponential distributions of the displacement are also found in glassy materials [58]. However, we have to stress that both situations are different since the needle can always diffuse along its long axis irrespective of the density and is only confined perpendicular to its long axis.

\section{Intermediate scattering function of the needle}

Information about the spatiotemporal dynamics of the needle center is encoded in the intermediate scattering function

$$
F(k, t)=\left\langle e^{-i \mathbf{k} \cdot \Delta \mathbf{r}(t)}\right\rangle=\int \mathrm{d}^{2} u \int \frac{\mathrm{d}^{2} u_{0}}{4 \pi} G_{k}\left(\mathbf{u}, t \mid \mathbf{u}_{0}\right),
$$

with wave vector $\mathbf{k}$ and wave number $k=|\mathbf{k}|$. Both integrals extend over all possible initial and final orientations. We have discussed the intermediate scattering function $F(k, t)$ recently for needle liquids as well as needle Lorentz systems and rationalized the results in terms of the phantom needle [26].

Here, we consider the intermediate scattering function in the case that all segments of the needle contribute to the scattering. For the two-dimensional analog where the needle moves in a planar array of point obstacles, the intermediate scattering function for the geometric center and the entire needle has been evaluated earlier and also compared to the phantom needle $[30,59]$.

We introduce the fluctuating density of the needle

$$
\rho_{\text {rod }}(\mathbf{k}, t)=\frac{1}{L} \int_{-L / 2}^{L / 2} \mathrm{~d} s \exp (\mathrm{ik} \cdot[\mathbf{r}(t)+s \mathbf{u}(t)]),
$$



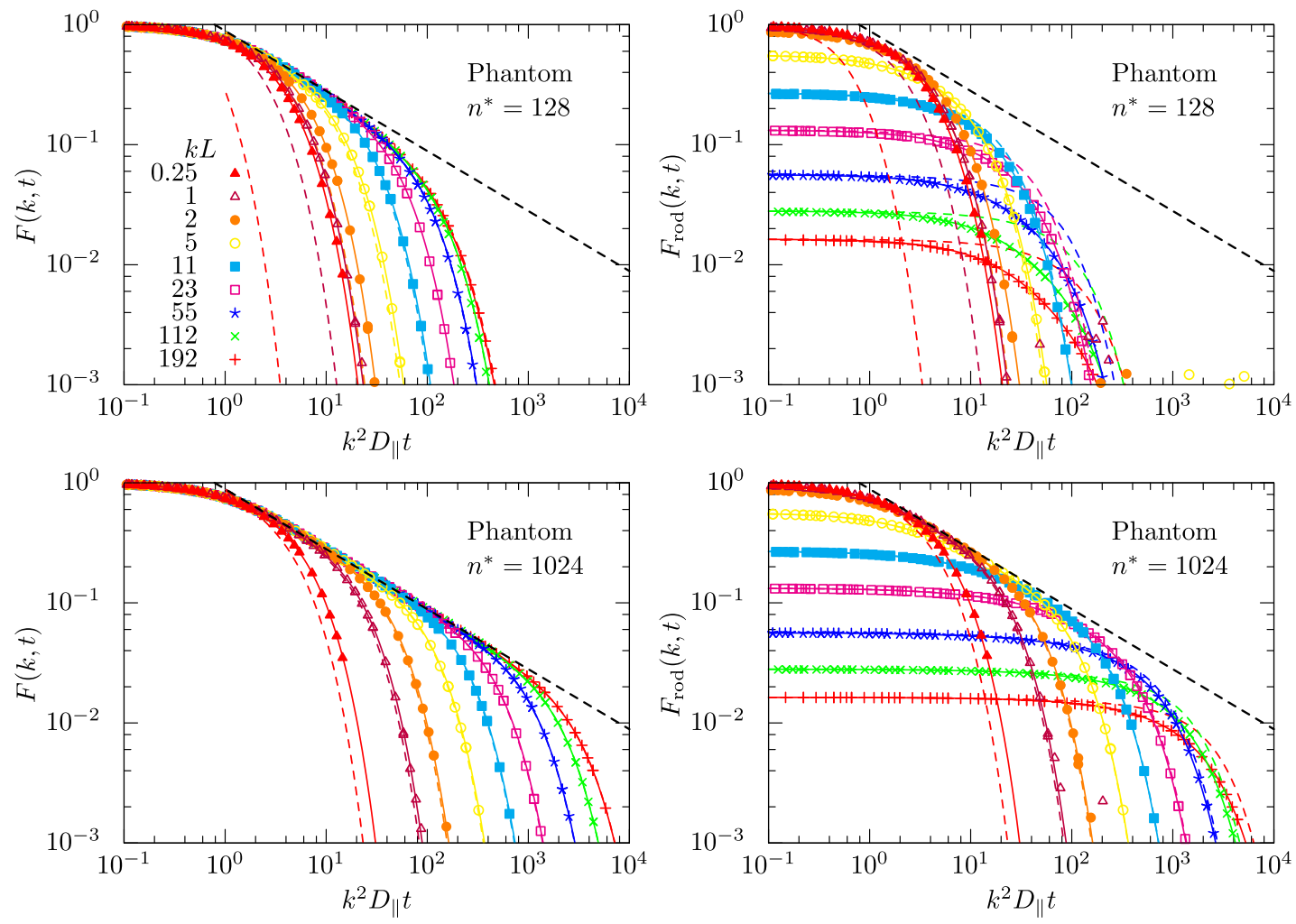

FIG. 7. Comparison of the intermediate scattering function of the center of mass, $F(k, t)$ (left), and of the whole needle, $F_{\text {rod }}(k, t)$ (right), of the phantom needle with transport coefficients from the needle Lorentz systems. Solid lines represent to the analytic solution and symbols correspond to simulation results of the phantom needle. The colored dashed lines represent the approximative solutions [Eqs. (35) and (36)] and the black dashed lines corresponds to the algebraic decay $\sim t^{-1 / 2}$ emerging from the sliding motion in the confining tube. The left panels are reproduced from Ref. [26].

where the integral extends over all segments $s$ of the needle. The corresponding intermediate scattering function is obtained via

$$
F_{\text {rod }}(k, t)=\left\langle\rho_{\text {rod }}(\mathbf{k}, t)^{*} \rho_{\text {rod }}(\mathbf{k}, 0)\right\rangle,
$$

where the angle brackets denote the same average as in the case of the intermediate scattering function of the needle center [Eq. (26)] and * is the complex conjugate. Thus, the quantity of interest is

$$
\begin{aligned}
& F_{\text {rod }}(k, t)=\frac{1}{L^{2}} \int \mathrm{d} s \int \mathrm{d} s_{0}\left\langle e^{-i \mathbf{k} \cdot \Delta \mathbf{r}(t)} e^{-i \mathbf{k} \cdot\left[s \mathbf{u}(t)-s_{0} \mathbf{u}(0)\right]}\right\rangle \\
& =\frac{1}{L^{2}} \int \mathrm{d} s \int \mathrm{d} s_{0} \int \mathrm{d}^{2} u \int \frac{\mathrm{d}^{2} u_{0}}{4 \pi} e^{-i \mathbf{k} \cdot\left[s \mathbf{u}-s_{0} \mathbf{u}_{0}\right]} G_{k}\left(\mathbf{u}, t \mid \mathbf{u}_{0}\right) .
\end{aligned}
$$

We follow the solution strategy in Ref. [40] and express the exponentials containing the segment of the needle in terms of the Rayleigh expansion,

$$
e^{\mathrm{i} k s z}=\sum_{l=0}^{\infty}(2 l+1) \mathrm{i}^{l} j_{l}(k s) \mathrm{P}_{l}(z),
$$

with spherical Bessel function $j_{l}(\cdot)$ and Legendre polynomial $\mathrm{P}_{l}(\cdot)$. Then, one can perform the average over all initial orientations $\mathbf{u}_{0}$ and integrate over all final orientations $\mathbf{u}$ with respect to the propagator $G_{k}$ [Eq. (7)]. Since the intermediate scattering function $F_{\text {rod }}(k, t)$ is isotropic, only spheroidal wave functions of order $m=0$ and even degree $n$ contribute and we expand the remaining spheroidal wave functions in Legendre polynomials via

$$
\operatorname{Ps}_{n}^{0}\left(z, \gamma^{2}\right)=\sum_{k=-\lfloor n / 2\rfloor}^{\infty}(-1)^{k} a_{n, k}^{0}\left(\gamma^{2}\right) \mathrm{P}_{n+2 k}(z) .
$$

Then, we obtain an expression suitable for numerical evaluation:

$$
F_{\text {rod }}(k, t)=\sum_{\substack{n=0 \\ n \text { even }}}^{\infty}(2 n+1) A_{n}^{2} e^{-\Gamma_{n}^{0} t} .
$$

The coefficients

$$
A_{n}=\sum_{\substack{l=0 \\ l \text { even }}}^{\infty}\left(a_{n,-(n-l) / 2}^{0}\right) \frac{1}{L} \int_{-L / 2}^{L / 2} \mathrm{~d} s j_{l}(k s)
$$

contain higher-order expansion coefficients $a_{n,-(n-l) / 2}^{0}$ of the spheroidal wave functions [Eq. (31)] which can be 

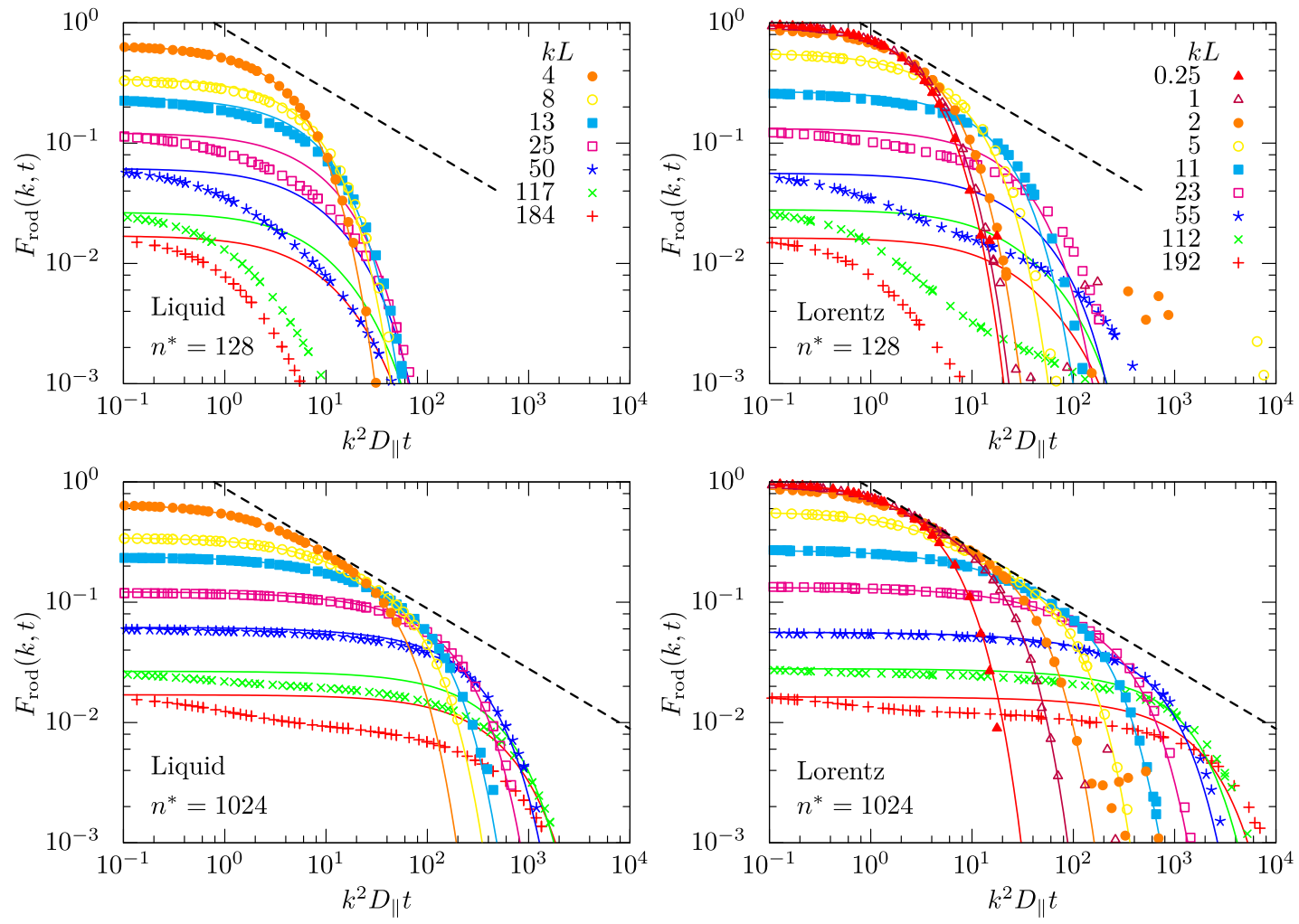

FIG. 8. Intermediate scattering function of the whole $\operatorname{rod} F_{\text {rod }}(k, t)$ at different densities $n^{*}$ for needle liquids as well as needle Lorentz systems. Symbols correspond to simulation results and solid lines represent the analytic solution [Eq. (32)] for the phantom needle. The black dashed lines corresponds to the algebraic decay $\sim t^{-1 / 2}$ emerging from the sliding motion in the confining tube. Wave number $k$ increases from top to bottom.

evaluated numerically [60, 61]. Moreover, only even orders $l$ have to be considered due to the symmetry property $j_{l}(-k s)=(-1)^{l} j_{l}(k s)$ of the spherical Bessel functions.

In computer simulations, we evaluate the intermediate scattering function via the formula

$$
F_{\text {rod }}(k, t)=\left\langle e^{-i \mathbf{k} \cdot \Delta \mathbf{r}(t)} f_{\mathbf{k}}(\mathbf{u}(t)) f_{\mathbf{k}}(\mathbf{u}(0))\right\rangle,
$$

with the form factor $f_{\mathbf{k}}(\mathbf{u})=\sin (\mathbf{k} \cdot \mathbf{u} L / 2) /(\mathbf{k} \cdot \mathbf{u} L / 2)$. The preceding equation is obtained after performing the integration over all segments in Eq. (29). The presence of the form factor in $F_{\text {rod }}(k, t)$ suggests that the scattering signal decorrelates faster than in $F(k, t)$ by the rotation of the needle.

First, we discuss both intermediate scattering functions $F(k, t)$ and $F_{\text {rod }}(k, t)$ for the phantom needle with transport coefficients obtained from the needle Lorentz system at densities $n^{*}=128$ and $n^{*}=1024$ [Fig. 7]. The full analytic solution for $F_{\text {rod }}(k, t)$ is corroborated by the simulation results for the phantom needle for all wave numbers and all times considered.

For small wave number $k L \lesssim 1$, both $F(k, t)$ and $F_{\text {rod }}(k, t)$ show similar time-dependent behavior since the dynamics is dominated by the diffusion of the geometric center of the needle [Fig. 7]. For increasing wave number, differences become apparent already at small times, since the intermediate scattering function of the geometric center is normalized, $F(k, 0)=1$, while the scattering function of the entire needle exhibits a static structure, i.e., a wave-number dependent initial value, $F_{\text {rod }}(k, 0)=$ $\int_{-1}^{1} \mathrm{~d} z j_{0}(k L z / 2)^{2} / 2=1-(k L)^{2} / 36+\mathcal{O}(k L)^{4}$.

At intermediate times, both scattering functions display a characteristic algebraic decay of the form $\sim t^{-1 / 2}$, which is a fingerprint of the sliding motion of the needle [33]. In particular, the intermediate scattering function for the geometric center, $F(k, t)$, can be evaluated in closed form for times $D_{\text {rot }} \gamma^{2} t \gtrsim 1$ and for wave numbers fulfilling $\gamma^{2}=k^{2} \Delta D / D_{\text {rot }} \gg 1$ in the highly entangled regime $D_{\text {rot }} \rightarrow 0$ to [26]

$$
F(k, t)=e^{-k^{2} D_{\perp} t} \sqrt{\frac{\pi}{\gamma}} \frac{1}{\sqrt{2 \sinh \left(2 D_{\operatorname{rot} \gamma t}\right)}} .
$$

The algebraic decay is observed for times $D_{\text {rot }} \gamma t \ll 1$ with $F(k, t)=1 / \sqrt{4 k^{2} \Delta D t / \pi}$ until the terminal relaxation $F(k, t)=\sqrt{(\pi / \gamma)} \exp \left[-\left(k^{2} D_{\perp}+D_{\text {rot }} \gamma\right) t\right]$ determines the time-dependent behavior for times $D_{\text {rot }} \gamma t \gg 1$ [Fig. 7].

With the full solution of $F_{\text {rod }}(k, t)$ [Eq. (32)] we can assess the validity of the approximate solution derived 
by Doi and Edwards for the highly entangled regime:

$$
F_{\text {rod }}(k, t)=e^{-k^{2} D_{\perp} t} \int_{-1}^{1} \mathrm{~d} z \frac{j_{0}(k L z / 2)^{2} e^{-\gamma z^{2} \tau_{-}}}{\sqrt{2 \sinh \left(2 D_{\text {rot }} \gamma t\right) \tau_{+}}},
$$

with $\tau_{ \pm}=\left[\cosh \left(2 D_{\text {rot }} \gamma t\right) \pm 1\right] / \sinh \left(2 D_{\text {rot }} \gamma t\right)$. In essence, the propagator $G_{k}$ [Eq. (6)] is approximated by that of a harmonic oscillator and the resulting average for the intermediate scattering function is evaluated for a needle which does not rotate significantly. As can be inferred from the comparison with the full solution [Fig. 7] the approximate solution is accurate for intermediate wave numbers, whereas deviations are present for small and high wave numbers. In particular, for high wave numbers the terminal relaxation is governed by a different prefactor of $\sqrt{(\gamma / 4 \pi)}\left[\int_{-1}^{1} \mathrm{~d} z j_{0}(k L z / 2) \exp \left(-\gamma z^{2} / 2\right)\right]^{2}$ not captured by the approximation [Eq. (36)].

In the presence of other needles [Fig. 8], the validity of the description of the dynamics in terms of the phantom needle depends on the density and the wave number under consideration. At density $n^{*}=128$, which marks the onset of the scaling behavior of the transport coefficients, the confining tube is only partially present and deviations become apparent already for wave numbers $k L \gtrsim 10$. Increasing the density by a factor of eight to $n^{*}=1024$, the phantom needle captures the dynamics at much smaller length scales until the dynamics within the tube is resolved for the largest wave numbers considered. For the needle Lorentz system at density $n^{*}=1024$, we also observe the characteristic algebraic decay $\sim t^{-1 / 2}$ in a small time window for the smallest wave numbers considered.

\section{SUMMARY AND CONCLUSION}

We have investigated the dynamics of solutions of infinitely thin needles for densities deep in the semidilute regime. The needles perform rotational and translational Brownian motion and are not allowed to cross each other.

From the time dependence of the rotational and translational diffusion, we have extracted the long-time diffusion coefficients and the geometry of the confining tube emerging for the high entanglement due to neighboring needles. The transport coefficients as well as the tilt angle and the tube diameter exhibit the predicted densitydependent scaling behavior obtained from theory and are observed in our simulation over one order of magnitude in the density.

Due to the strong suppression of the rotational and perpendicular translational diffusion coefficients, the mean-square displacement of the geometric center on coarse-grained time scales for the strong entanglement is purely determined by the diffusion coefficient for the motion parallel to the long axis. For the non-Gaussian parameter, we observe the pure sliding motion in the confining tube as an intermediate plateau over many decades in time.
An analytic expression for the intermediate scattering function of the entire needle has been derived and evaluated numerically. In comparison to the intermediate scattering function of the geometric center, the characteristic algebraic decay corresponding to the sliding within the tube is much less pronounced and only observed in a small time window and for the smallest wave numbers in the high entanglement.

On coarse-grained time and length scales, the phantom needle with long-time translational and rotational diffusion coefficients as input parameters captures the dynamics of the needles in solution for all the considered quantities as anticipated from the tube model of Doi and Edwards. We also performed simulations on needle Lorentz systems where a single tracer needle performs Brownian motion in a quenched array of other needles. The dynamics in needle Lorentz systems is identical to the dynamics in needle liquids and the dynamic rearrangement due to motion of the other needles only admits a change in the absolute values of the long-time diffusion coefficients.

Here, we have considered the transport properties of monodisperse solutions of needles. It would be interesting to go beyond this model and consider the diffusion of a tracer needle of length $L_{\mathrm{t}}$ in a solution (matrix) of needles of a different length $L_{\mathrm{s}}$. For needles which perform translational motion only, the scaling behavior of the perpendicular translational diffusion coefficient of the tracer has been elaborated theoretically [43] and a different behavior of $D_{\perp} \sim\left(n^{*}\right)^{-1}$ is predicted if the needles of the matrix are much shorter than the tracer needle, $L_{\mathrm{s}} \ll L_{\mathrm{t}}$. In the opposite case $L_{\mathrm{t}} \ll L_{\mathrm{s}}$, where the matrix evolves much slower than the tracer needle which is reminiscent of a needle Lorentz system, one theoretically recovers the scaling behavior $D_{\perp} \sim\left(n^{*}\right)^{-2}$. Although both cases are computationally more demanding since the averages are performed for the tracer needle only, it should in principle be possible to assess these predictions in our simulation. Moreover, one can consider the dynamics also in the presence of additional spherical particles which affect the diffusion of the needle parallel to their long axis [62].

In the case of hard rods of finite diameter $b[22,23,63]$ the semidilute regime extends up to densities of $n \ll$ $1 / b L^{2}$ where the excluded volume becomes relevant and influences the dynamics. As long as the diameter of the rod is much smaller than its length $b \ll L$, the scaling behavior of the rotational diffusion coefficient in the semidilute regime is still governed by the same scaling law $[16,22,23]$. For the additional quantities considered here, one anticipates that the phantom needle still captures the dynamics of such very elongated hard rod solutions on coarse-grained time and length scales.

One may also relax the requirement of stiff fibers and consider the dynamics in the case of semiflexible polymers [64-67]. While the tube concept still provides the key insight into the dynamics of such solutions in the highly entangled regime [68, 69], the tube displays additional tube-width fluctuations $[56,57,70]$ and the tube renewal becomes more complex which directly affects the 
behavior of the transport coefficients. However, tubewidth fluctuations are not only important for semiflexible polymers, but also appear to be a relevant concept for needle solutions as has been shown just recently [45, 71].

Our simulations of needle Lorentz systems and needle liquids deep in the semidilute regime heavily rely on the use of a geometry-adapted neighbor list which significantly reduces the computational time for the collision detection. Such neighbor lists have been considered before for nonspherical particles [72] and the obtained speedup for the simulation should at least in part be transferable to simulations of hard semiflexible polymers.

\section{Appendix A: Simulation of the hard-core interaction between needles}

We employ a pseudo-Brownian scheme to describe the hard-core interaction between the needles [36, 37]. The scheme builds on an event-based algorithm to propagate the needle within one Brownian time step. It extends a collision detection algorithm developed earlier for a needle moving in a two-dimensional array of point obstacles [73]. We always move only a single needle at a time such that the formulas simplify. For the general case, we refer to Ref. [74].

During a Brownian time step $\Delta t \in\left[0, \tau_{\mathrm{B}}\right]$, the needle rotates in a plane perpendicular to the rotational pseudovelocity $\boldsymbol{\omega}$, and the geometric center $\mathbf{r}(t+\Delta t)=$ $\mathbf{r}(t)+\mathbf{v} \Delta t$ located in the rotational plane moves with pseudotranslational velocity $\mathbf{v}$. A collision candidate needle of the same length $L$ with position $\mathbf{r}_{c}$ and orientation $\mathbf{u}_{c}$ intersects the rotational plane of the moving needle for times for which the following inequality holds:

$$
\left|\boldsymbol{\omega} \cdot \Delta \mathbf{r}_{c}(t+\Delta t)\right| \leq \frac{L}{2}\left|\boldsymbol{\omega} \cdot \mathbf{u}_{c}\right|
$$

where we defined the distance $\Delta \mathbf{r}_{c}(t+\Delta t)=\mathbf{r}(t+\Delta t)-\mathbf{r}_{c}$ of the geometric needle centers.

In addition to that, the intersection point $\mathbf{r}_{I}(t+\Delta t)$ of the collision candidate needle with the rotational plane has to traverse the rotational disk of radius $L / 2$ of the moving needle. Defining the distance vector $\Delta \mathbf{r}_{\mathrm{pl}}(t+\Delta t)=\mathbf{r}(t+\Delta t)-\mathbf{r}_{I}(t+\Delta t)$ of the geometric center of the moving needle with the intersection point in the rotational plane, the relevant time interval is determined by the inequality

$$
\left[\Delta \mathbf{r}_{\mathrm{pl}}(t+\Delta t)\right]^{2} \leq\left(\frac{L}{2}\right)^{2}
$$

Both of the preceding conditions [Eqs. (A1) and (A2)] define a smaller time interval $\left[\tau_{l}, \tau_{u}\right] \subset\left[0, \tau_{\mathrm{B}}\right]$, which has to be nonempty for a possible collision of both needles. A necessary condition for a collision at times $\Delta t \in\left[\tau_{l}, \tau_{u}\right]$ is that the distance vector $\Delta \mathbf{r}_{\mathrm{pl}}(t+\Delta t)$ and the orientation $\mathbf{u}(t+\Delta t)$ of the moving needle become parallel:

$$
\boldsymbol{\omega} \cdot\left[\Delta \mathbf{r}_{\mathrm{pl}}(t+\Delta t) \times \mathbf{u}(t+\Delta t)\right]=0 .
$$

The scalar product with the pseudorotational velocity $\boldsymbol{\omega}$ is used both to obtain a one-dimensional equation and to enforce a change of sign at the root. In order to determine the collision time and also if a collision even occurs, we use an interval Newton method [28, 73, 75] on the time interval $\left[\tau_{l}, \tau_{u}\right]$. In order not to miss collisions, a correctly rounded math library [76] has to be used for the transcendental functions in the change of orientation, $\mathbf{u}(t+\Delta t)[\mathrm{Eq} .(2)]$. This procedure yields the point in time $t+\tau_{\mathrm{c}}$ of the next collision.

Upon colliding at time $t+\tau_{\mathrm{c}}$, the moving needle acquires new velocities for rotation and translation which are determined by conservation of energy, momentum, and angular momentum. Here, we assume that the center of mass of the moving needle coincides with its geometric center and consider only smooth needles [74, 77], where the momentum transfer $\Delta \mathbf{p}=\Delta p \mathbf{e}_{m}$ is perpendicular to the orientation of both collision partners and directed along $\mathbf{e}_{m}=\mathbf{u}\left(t+\tau_{c}\right) \times \mathbf{u}_{c} /\left|\mathbf{u}\left(t+\tau_{c}\right) \times \mathbf{u}_{c}\right|$. The magnitude of the momentum transfer, $\Delta p$, depends on the translational and rotational velocities $\mathbf{v}$ and $\boldsymbol{\omega}$, respectively, and on the point of contact $\mathbf{r}_{\mathrm{coll}}=\Delta \mathbf{r}_{\mathrm{pl}}\left(t+\tau_{\mathrm{c}}\right)$ with respect to the center of the moving needle:

$$
\Delta p=-2 \frac{\mathbf{v} \cdot \mathbf{e}_{m}+\boldsymbol{\omega} \cdot\left(\mathbf{r}_{\mathrm{coll}} \times \mathbf{e}_{m}\right)}{1+\frac{m}{I}\left(\mathbf{r}_{\mathrm{coll}} \times \mathbf{e}_{m}\right)^{2}}
$$

Then, the new velocities $\mathbf{v}^{\prime}$ and $\boldsymbol{\omega}^{\prime}$ for translation and rotation for the remaining Brownian time interval $\left[\tau_{\mathrm{c}}, \tau_{\mathrm{B}}\right]$ are determined by

$$
\begin{aligned}
\mathbf{v}^{\prime} & =\mathbf{v}+\Delta p \mathbf{e}_{m}, \\
\boldsymbol{\omega}^{\prime} & =\boldsymbol{\omega}+\frac{m}{I} \Delta p\left(\mathbf{r}_{\text {coll }} \times \mathbf{e}_{m}\right) .
\end{aligned}
$$

The ratio of mass $m$ and inertia $I$ can be related to the short-time diffusion coefficients by prohibiting an average flow of energy between the rotational and translational degrees of freedom of the moving needle. We define pseudotemperatures $T_{\text {rot }}$ and $T_{\perp}$ via the relations $I\left\langle\boldsymbol{\omega}^{2}\right\rangle / 2=2 k_{\mathrm{B}} T_{\text {rot }} / 2$ and $m\left\langle\mathbf{v}_{\perp}^{2}\right\rangle=2 k_{\mathrm{B}} T_{\perp} / 2$ and determine the remaining averages as $\left\langle\boldsymbol{\omega}^{2}\right\rangle=4 D_{\text {rot }}^{0} / \tau_{\mathrm{B}}$ and $\left\langle\mathbf{r}_{\perp}^{2}\right\rangle=4 D_{\perp}^{0} / \tau_{\mathrm{B}}$ from the equations for the pseudovelocities [Eqs. (3)]. Then, for $T_{\text {rot }}=T_{\perp}$, the average flow of energy at collision vanishes and we obtain

$$
\frac{m}{I}=\frac{D_{\mathrm{rot}}^{0}}{D_{\perp}^{0}}
$$

For anisotropic particles such as needles, it is possible that the moving needle collides again with the previous collision partner in the remaining time interval $\left[\tau_{\mathrm{c}}, \tau_{\mathrm{B}}\right]$. A minimal propagation time for such an event can be estimated by considering the moving needle and the intersection point of the collision partner in the new rotational plane determined by $\boldsymbol{\omega}^{\prime}$. Ignoring the length of both needles, the minimal collision time $\tau_{\mathrm{r}}$ for a repeated collision is obtained as a solution of the transcendental 
equation

$$
\frac{\omega^{\prime} \tau_{\mathrm{r}}}{\tan \left(\omega^{\prime} \tau_{\mathrm{r}}\right)}=\omega^{\prime} \frac{\left[\Delta \mathbf{r}_{\mathrm{pl}}\left(t+\tau_{\mathrm{c}}\right)+\mathbf{v}_{\mathrm{pl}}^{\prime} \tau_{\mathrm{r}}\right] \cdot \mathbf{u}\left(t+\tau_{\mathrm{c}}\right)}{\mathbf{v}_{\mathrm{pl}}^{\prime} \cdot\left[\boldsymbol{\omega}^{\prime} / \omega^{\prime} \times \mathbf{u}\left(t+\tau_{\mathrm{c}}\right)\right]},
$$

with the velocity of the intersection point in the rotational plane $-\mathbf{v}_{\mathrm{pl}}^{\prime}=-\mathbf{v}^{\prime}+\left(\boldsymbol{\omega}^{\prime} \cdot \mathbf{v}^{\prime}\right) \mathbf{u}_{c} /\left(\boldsymbol{\omega}^{\prime} \cdot \mathbf{u}_{c}\right)$. By considering the series expansion for both $\tan (\cdot)$ and $\cot (\cdot)$, the function on the left hand side can be estimated by the inequality $1-\left(\omega^{\prime} \tau_{\mathrm{r}}\right)^{2} / 2 \leq \omega^{\prime} \tau_{\mathrm{r}} / \tan \left(\omega^{\prime} \tau_{\mathrm{r}}\right) \leq 1-\left(\omega^{\prime} \tau_{\mathrm{r}}\right)^{2} / 3$ valid for times $0 \leq \omega^{\prime} \tau_{\mathrm{r}}<\pi / 2$. Hence, by solving for two quadratic equations, we obtain a lower bound for the minimal collision time $\tau_{\mathrm{r}}$ and only choose $\omega^{\prime} \tau_{\mathrm{r}}=\pi / 2$ if the solutions are located outside of the validity of the approximation.

To determine all possible collision candidate needles, we enclose the moving needle in a fixed cylinder, which is valid as long as the moving needle does not touch the boundary. Then, all needles intersecting the cylinder belong to the class of collision candidates. The optimal size of the cylinder has to be determined via the simulation and decreases with decreasing Brownian time $\tau_{\mathrm{B}}$. For liquid configurations where we move every needle subsequently, we consider the intersection of the corresponding cylinders. This geometry-adapted neighbor list significantly reduces the computational time in the dense regime.

The collision detection is further supplemented by a simple test performed on the time interval $\left[\tau_{l}, \tau_{u}\right]$ obtained after checking for both length conditions [Eqs. (A1) and (A2)]. During time $\left[0, \tau_{u}\right]$ the needle covers two sectors of angle $\omega \tau_{u}$ and we approximate the area by two lines parallel to $\mathbf{u}(t)$ in the rotational plane with minimal distance $L \sin \left(\omega \tau_{u}\right) / 2$ to the geometric center of the moving needle. Then, a collision can only happen if the intersection point traverses this area in the interval $\left[\tau_{l}, \tau_{u}\right]$.

\section{Appendix B: Moments of the needle displacement}

Since the intermediate scattering function of the geometric center, $F(k, t)=\left\langle e^{-\mathbf{i k} \cdot \Delta \mathbf{r}(t)}\right\rangle[\mathrm{Eq} \cdot(26)]$, is isotropic in the wave vector $\mathbf{k}$, we average over all possible orientations of the wave vector and obtain an expression which manifestly displays the symmetry:

$$
F(k, t)=\left\langle\frac{\sin (k|\Delta \mathbf{r}(t)|)}{k|\Delta \mathbf{r}(t)|}\right\rangle,
$$

with wave number $k=|\mathbf{k}|$. Then, the mean-square displacement $\operatorname{MSD}(t)$ and the mean-quartic displacement $\operatorname{MQD}(t)$ of the needle center are encoded in the smallwave-number behavior

$$
F(k, t)=1-\frac{k^{2}}{3 !} \operatorname{MSD}(t)+\frac{k^{4}}{5 !} \operatorname{MQD}(t)+\mathcal{O}\left(k^{6}\right) .
$$

Since the full solution of the intermediate scattering function can be expressed in terms of spheroidal wave functions via

$$
F(k, t)=\sum_{\substack{n=0 \\ n \text { even }}}^{\infty} \frac{2 n+1}{4}\left[\int_{-1}^{1} \mathrm{~d} z \operatorname{Ps}_{n}^{0}\left(z, \gamma^{2}\right)\right]^{2} e^{-\Gamma_{n}^{0} t}
$$

with decay constants $\Gamma_{n}^{0}=D_{\|} k^{2}+D_{\text {rot }} \lambda_{n}^{0}$, we use a time-independent perturbation theory in the wave number $k$, similar to Ref. [55]. We derive the dependence of the spheroidal eigenvalue $\lambda_{n}^{0}\left(\gamma^{2}\right)$ and the spheroidal wave function on the real parameter $\gamma^{2}$ up to order $\mathcal{O}\left(\gamma^{4}\right)$. For the mean-square and the mean-quartic displacement only $n=0$ and $n=2$ in the intermediate scattering function $F(k, t)$ [Eq. (B3)] contribute and the expansion for the spheroidal eigenvalue $\lambda_{n}^{0}$ reads [78]

$\lambda_{n}^{0}\left(\gamma^{2}\right)-n(n+1)= \begin{cases}-\frac{2}{3} \gamma^{2}-\frac{2}{135} \gamma^{4}+\mathcal{O}\left(\gamma^{6}\right), & n=0, \\ -\frac{10}{21} \gamma^{2}+\frac{94}{9261} \gamma^{4}+\mathcal{O}\left(\gamma^{6}\right), & n=2 .\end{cases}$

Similarly for the integral of the spheroidal wave function we obtain

$$
\int_{-1}^{1} \mathrm{~d} z \operatorname{Ps}_{n}^{0}\left(z, \gamma^{2}\right)= \begin{cases}2-\frac{1}{405} \gamma^{4}+\mathcal{O}\left(\gamma^{6}\right), & n=0 \\ \frac{2}{45} \gamma^{2}-\frac{4}{2835} \gamma^{4}+\mathcal{O}\left(\gamma^{6}\right), & n=2\end{cases}
$$

Then, both moments can be obtained by comparing the resulting expression to the small-wave number behavior $[\mathrm{Eq} .(\mathrm{B} 2)]$.

\section{ACKNOWLEDGMENTS}

The computational results presented have been achieved (in part) using the HPC infrastructure LEO of the University of Innsbruck. We acknowledge financial support by the Deutsche Forschungsgemeinschaft (DFG) Contract No. FR1418/5-1.
[1] B. Hinner, M. Tempel, E. Sackmann, K. Kroy, and E. Frey, Phys. Rev. Lett. 81, 2614 (1998).
[2] I. Y. Wong, M. L. Gardel, D. R. Reichman, E. R. Weeks, M. T. Valentine, A. R. Bausch, and D. A. Weitz, Phys. 
Rev. Lett. 92, 178101 (2004).

[3] J. Liu, M. L. Gardel, K. Kroy, E. Frey, B. D. Hoffman, J. C. Crocker, A. R. Bausch, and D. A. Weitz, Phys. Rev. Lett. 96, 118104 (2006).

[4] G. H. Koenderink, M. Atakhorrami, F. C. MacKintosh, and C. F. Schmidt, Phys. Rev. Lett. 96, 138307 (2006).

[5] Y.-C. Lin, G. H. Koenderink, F. C. MacKintosh, and D. A. Weitz, Macromolecules 40, 7714 (2007).

[6] G. H. Koenderink, S. Sacanna, D. G. A. L. Aarts, and A. P. Philipse, Phys. Rev. E 69, 021804 (2004).

[7] M. P. Lettinga and E. Grelet, Phys. Rev. Lett. 99, 197802 (2007).

[8] E. Grelet, Phys. Rev. X 4, 021053 (2014).

[9] P. Cassagnau, W. Zhang, and B. Charleux, Rheol. Acta 52, 815 (2013).

[10] A. R. Bausch and K. Kroy, Nat. Phys. 2, 231 (2006).

[11] M. J. Solomon and P. T. Spicer, Soft Matter 6, 1391 (2010).

[12] Y. Han, A. M. Alsayed, M. Nobili, J. Zhang, T. C. Lubensky, and A. G. Yodh, Science 314, 626 (2006).

[13] R. Duggal and M. Pasquali, Phys. Rev. Lett. 96, 246104 (2006).

[14] D. Mukhija and M. J. Solomon, Journal of Colloid and Interface Science 314, 98 (2007).

[15] M. Doi and S. F. Edwards, The Theory of Polymer Dynamics (Oxford University Press, Oxford, 1999).

[16] M. Doi, J. Phys. (France) 36, 607 (1975).

[17] I. Teraoka, N. Ookubo, and R. Hayakawa, Phys. Rev. Lett. 55, 2712 (1985).

[18] I. Teraoka and R. Hayakawa, J. Chem. Phys. 89, 6989 (1988).

[19] I. Teraoka and R. Hayakawa, J. Chem. Phys. 91, 2643 (1989).

[20] G. Szamel, Phys. Rev. Lett. 70, 3744 (1993).

[21] M. Doi, I. Yamamoto, and F. Kano, J. Phys. Soc. Jpn. 53, 3000 (1984).

[22] P. D. Cobb and J. E. Butler, J. Chem. Phys. 123, 054908 (2005).

[23] Y.-G. Tao, W. K. den Otter, J. K. G. Dhont, and W. J. Briels, J. Chem. Phys. 124, 134906 (2006).

[24] Y.-L. S. Tse and H. C. Andersen, J. Chem. Phys. 139, 044905 (2013).

[25] T. Zhao and X. Wang, Polymer 54, 5241 (2013).

[26] S. Leitmann, F. Höfling, and T. Franosch, Phys. Rev. Lett. 117, 097801 (2016).

[27] A. J. Moreno and W. Kob, Europhys. Lett. 67, 820 (2004).

[28] F. Höfling, E. Frey, and T. Franosch, Phys. Rev. Lett. 101, 120605 (2008).

[29] F. Höfling, T. Munk, E. Frey, and T. Franosch, Phys. Rev. E 77, 060904 (2008).

[30] T. Munk, F. Höfling, E. Frey, and T. Franosch, Europhys. Lett. 85, 30003 (2009).

[31] A. K. Tucker and R. Hernandez, J. Phys. Chem. A 114, 9628 (2010).

[32] D. Kasimov, T. Admon, and Y. Roichman, Phys. Rev. E 93, 050602 (2016).

[33] M. Doi and S. F. Edwards, J. Chem. Soc., Faraday Trans. 2 74, 560 (1978).

[34] G. S. Chirikjian, Stochastic Models, Information Theory, and Lie Groups, Vol. 1 (Birkhäuser, Boston, 2009).

[35] B. Oksendal, Stochastic Differential Equations: An Introduction with Applications, 6th ed. (Springer, Berlin, 2010).
36] A. Scala, Th. Voigtmann, and C. De Michele, J. Chem. Phys. 126, 134109 (2007).

[37] F. Höfling, T. Munk, E. Frey, and T. Franosch, The Journal of Chemical Physics 128, 164517 (2008).

[38] J. K. Dhont and W. Briels, Colloids and Surfaces A: Physicochemical and Engineering Aspects 213, 131 (2003).

[39] B. J. Berne and R. Pecora, Dynamic Light Scattering: With Applications to Chemistry, Biology, and Physics (Dover Publications, Meniola, 2000).

[40] S. R. Aragón S. and R. Pecora, J. Chem. Phys. 82, 5346 (1985).

[41] DLMF, "NIST digital library of mathematical functions," http://dlmf.nist.gov/, Release 1.0.10 of 2015-0807 , online companion to [42].

[42] F. W. J. Olver, D. W. Lozier, R. F. Boisvert, and C. W. Clark, eds., NIST Handbook of Mathematical Functions (Cambridge University Press, New York, NY, 2010) print companion to [41].

[43] G. Szamel and K. S. Schweizer, J. Chem. Phys. 100, 3127 (1994).

[44] D. M. Sussman and K. S. Schweizer, Phys. Rev. E 83 , 061501 (2011).

[45] D. M. Sussman and K. S. Schweizer, Phys. Rev. Lett. 107, 078102 (2011).

[46] T. O. E. Skinner, S. K. Schnyder, D. G. A. L. Aarts, J. Horbach, and R. P. A. Dullens, Phys. Rev. Lett. 111, 128301 (2013).

[47] S. K. Schnyder, M. Spanner, F. Höfling, T. Franosch, and J. Horbach, Soft Matter 11, 701 (2015).

[48] M. Spanner, F. Höfling, S. C. Kapfer, K. R. Mecke, G. E. Schröder-Turk, and T. Franosch, Phys. Rev. Lett. 116, 060601 (2016).

[49] Th. Voigtmann and J. Horbach, Phys. Rev. Lett. 103, 205901 (2009).

[50] J. Horbach, Th. Voigtmann, F. Höfling, and T. Franosch, Eur. Phys. J. Special Topics 189, 141 (2010).

[51] W. Götze, Complex Dynamics of Glass-Forming Liquids: A Mode-Coupling Theory (Oxford University Press, Oxford, 2012).

[52] A. Rahman, Phys. Rev. 136, A405 (1964).

[53] F. Höfling and T. Franosch, Rep. Prog. Phys. 76, 046602 (2013).

[54] M. Dibak, Brownian motion of optically trapped ellipsoids, Bachelor thesis, Universität Stuttgart, Stuttgart (2013).

[55] C. Kurzthaler, S. Leitmann, and T. Franosch, Scientific Reports 6, 36702 (2016).

[56] B. Wang, J. Guan, S. M. Anthony, S. C. Bae, K. S. Schweizer, and S. Granick, Phys. Rev. Lett. 104, 118301 (2010).

[57] J. Glaser, D. Chakraborty, K. Kroy, I. Lauter, M. Degawa, N. Kirchgeßner, B. Hoffmann, R. Merkel, and M. Giesen, Phys. Rev. Lett. 105, 037801 (2010).

[58] P. Chaudhuri, L. Berthier, and W. Kob, Phys. Rev. Lett. 99, 060604 (2007).

[59] T. Munk, Complex Transport Processes in Suspensions of Stiff Polymers, Ph.D. thesis, Ludwig-MaximiliansUniversität München (2008).

[60] D. B. Hodge, J. Math. Phys. 11, 2308 (1970).

[61] P. E. Falloon, P. C. Abbott, and J. B. Wang, J. Phys. A 36, 5477 (2003).

[62] U. Yamamoto and K. S. Schweizer, ACS Macro Lett. 4, $53(2015)$ 
[63] H. Löwen, Phys. Rev. E 50, 1232 (1994).

64] D. C. Morse, Phys. Rev. E 63, 031502 (2001).

[65] S. Ramanathan and D. C. Morse, Phys. Rev. E 76, 010501 (2007).

[66] G. Nam, A. Johner, and N.-K. Lee, J. Chem. Phys. 133, 044908 (2010).

[67] S. A. Egorov, A. Milchev, and K. Binder, Phys. Rev. Lett. 116, 187801 (2016).

[68] T. Odijk, Macromolecules 16, 1340 (1983).

[69] A. N. Semenov, J. Chem. Soc., Faraday Trans. 2 82, 317 (1986).

[70] J. Glaser and K. Kroy, Phys. Rev. E 84, 051801 (2011).

[71] D. M. Sussman and K. S. Schweizer, Phys. Rev. Lett. 109, 168306 (2012).

[72] A. Donev, S. Torquato, and F. H. Stillinger, J. Comput. Phys. 202, 737 (2005).
[73] F. Höfling, Dynamics of Rod-Like Macromolecules in Heterogeneous Materials, Ph.D. thesis, LudwigMaximilians-Universität München (2006).

[74] D. Frenkel and J. F. Maguire, Mol. Phys. 49, 503 (1983).

[75] E. Hansen and G. W. Walster, Global Optimization Using Interval Analysis, 2nd ed. (Marcel Dekker, New York, 2004).

[76] C. Daramy-Loirat, D. Defour, F. de Dinechin, M. Gallet, N. Gast, C. Q. Lauter, and J.-M. Muller, "CR-LIBM: A Library of Corretly Rounded Elementary Functions in Double-Precision," (2009).

[77] M. Otto, T. Aspelmeier, and A. Zippelius, J. Chem. Phys. 124, 154907 (2006).

[78] J. Meixner and F. W. Schäfke, Mathieusche Funktionen und Sphäroidfunktionen (Springer, Berlin, 1954). 